\title{
Pharmacokinetic and Pharmacodynamic Evaluation of Nasal Liposome and Nanoparticle Based Rivastigmine Formulations in Acute and Chronic Models of Alzheimer's Disease
}

\section{Sampath Kumar L Rompicherla}

Manipal College of Pharmaceutical Sciences

Karthik Arumugam

Manipal College of Pharmaceutical Sciences

Sree Lalitha Bojja

Manipal College of Pharmaceutical Sciences

Nitesh Kumar

NIPER Hajipur: National Institute of Pharmaceutical Education and Research Hajipur

Mallikarjuna Rao Chamallamudi ( $\sim$ mallik.rao@manipal.edu )

Manipal College of Pharmaceutical Sciences

\section{Research Article}

Keywords: PK-PD modelling, Amnesia, Neurodegeneration, Nanoformulation, Nose to brain delivery

Posted Date: February 10th, 2021

DOI: https://doi.org/10.21203/rs.3.rs-181863/v1

License: (c) (1) This work is licensed under a Creative Commons Attribution 4.0 International License.

Read Full License 


\section{Abstract}

With the increasing ageing population and progressive nature of the disease, Alzheimer's disease (AD) poses to be an oncoming epidemic with limited therapeutic strategies. It is characterized by memory loss, behavioral instability, impaired cognitive function, predominantly, cognitive inability manifested due to the accumulation of $\beta$-amyloid, with malfunctioned cholinergic system. Rivastigmine, a reversible dual cholinesterase inhibitor is more tolerable and widely used choice of drug for AD. However, rivastigmine being hydrophilic and undergoing first pass metabolism, exhibits low CNS bioavailability. Nanoformulations including liposomes and PLGA nanoparticles can encapsulate hydrophilic drugs and deliver efficiently to brain. Besides, the nasal route is receiving considerable attention recently, due to its direct access to brain. Therefore, the present study attempts to evaluate the pharmacokinetic and pharmacodynamic properties of nasal liposomal and PLGA nanoparticle formulations of rivastigmine in scopolamine induced amnesia model and validate the best formulation by employing pharmacokinetic and pharmacodynamic (PK-PD) modelling. Nasal liposomal rivastigmine formulation showed the best pharmacokinetic features with rapid onset of action (Tmax=5 minutes), higher Cmax (1489.5 \pm 620.71$)$, enhanced systemic bioavailability $(F=118.65 \pm 23.54$; AUC $=35921.75 \pm 9559.46)$, increased half-life (30.92 \pm 8.38 minutes), and reduced clearance rate $(\operatorname{Kel}(1 / \mathrm{min})=0.0224 \pm 0.006)$ compared to oral rivastigmine $(\operatorname{Tmax}=15$ minutes; $\mathrm{Cmax}=56.29 \pm 27.05 ; \mathrm{F}=4.39 \pm 1.82 ; \mathrm{AUC}=1663.79 \pm 813.54 ; \mathrm{t} 1 / 2=$ $13.48 \pm 5.79 ; \mathrm{Kel}(1 / \mathrm{min})=0.0514 \pm 0.023)$. Further, the liposomal formulation significantly rescued the memory deficit induced by scopolamine superior to other formulations as assessed in Morris water maze and passive avoidance tasks. PK-PD modelling demonstrated strong correlation between the pharmacokinetic parameters and acetylcholinesterase inhibition of liposomal formulation.

\section{Introduction}

With the increasing ageing population and progressive nature of the disease, Alzheimer's disease (AD) poses to be an oncoming epidemic with limited therapeutic strategies [1, 2]. AD is the commonest cause of dementia, and dementia constitutes the fifth leading cause of death globally [3]. It is characterized by deposition of extracellular amyloid $\beta$ plaques and intracellular tau neurofibrillary tangles, which leads to neuroinflammation, synaptic dysfunction and subsequently, progressive neurodegeneration [4]. Lately, the drug pipeline for AD remains dry with failed clinical trials and no new FDA approved drugs since 2003. The existing few therapeutic drugs, rivastigmine and donepezil can only alleviate the symptoms [5].

Degeneration of cholinergic neurons and resulting loss in cholinergic transmission promotes the cognitive decline in AD patients. Cholinesterase inhibitors represent the significant fraction of currently available drugs as they primarily enhance the cholinergic neurotransmission in the brain by delaying the degradation of acetylcholine available in the synaptic clefts [5]. Among the FDA approved cholinesterase inhibitors, Donepezil, galantamine and rivastigmine, rivastigmine is superior and widely used, owing to its enhanced specificity and low risk of adverse effects. It is a second-generation carbamate derivative and reversible, noncompetitive acetyl- and butyryl-cholinesterase inhibitor. It is widely used in mild to moderate AD cases, and studies propose maximal therapeutic benefits with early and continuous 
treatment. Recommended doses for improving the cognitive symptoms is $6-12 \mathrm{mg} /$ day, b.i.d, p.o. It has high CNS selectivity, however has poor BBB penetration with log P predicted as 2.45 . In addition, it has low bioavailability of $36-40 \%$ due to its hydrophilic nature as well as the extensive first-pass metabolism leading to short plasma elimination half-life $(1.5 \mathrm{~h})$ [6]. Lately developed transdermal patch of rivastigmine has overcome some of its shortcomings, however, brain penetration is still poorer [7]. Therefore, alternative brain targeted delivery strategies are the need of the hour.

Intranasal drug delivery, a non-invasive approach has gained immense interest recently because of its direct transport of drugs to brain through olfactory and trigeminal nerve pathways, circumventing the brain barriers. Further, this route provides rapid onset of action besides avoiding the gastrointestinal breakdown and first pass metabolism [8]. Thus, the nasal route can be exploited in the present study to deliver rivastigmine efficiently to the brain. Despite the direct access to the brain, limited drug absorption, and nasal permeability remains challenge with nasal delivery of hydrophilic drugs. To overcome this, nanocarriers such as PLGA nanoparticles and liposomes represent efficient vehicle systems to deliver the hydrophilic cargo (rivastigmine) to brain [9]. Therefore, the present study aims to evaluate the pharmacokinetic and pharmcodynamic profile of liposomal and nanoparticle formulations of rivastigmine delivered nasally to treat dementia.

\section{Materials And Methods \\ 2.1. Animals}

The study was performed on healthy male Wistar rats aged 10 months, weighing $275 \pm 10 \mathrm{~g}$ procured from the central animal house research facility, Manipal University, Manipal. Based on the earlier report of effect of gender on the pharmacokinetics of rivastigmine, males were chosen for the study [10]. All experiments were performed as per the guidelines of the Institutional Animal ethical committee of Manipal University. Rats were housed in polyacrylic cage in the animal house at controlled conditions temperature $22 \pm 2{ }^{\circ} \mathrm{C}$, humidity $60 \pm 2 \%$ and 12-hour diurnal light cycle. Water and food in the form of dry pellets were provided ad libitum. All behavioral experiments were carried out from 18 to 21 hours of the day in a room adjacent to that in which the rats were housed.

\subsection{Chemicals}

Acetylthiocholine iodide (ATCI), scopolamine hydrobromide, and colchicine were purchased from Sigma Chemicals co., USA. Rivastigmine hydrogen tartrate (99\%) was obtained as a gift sample from Dr. Reddy's Laboratories Limited, Hyderabad, India. Venlafaxine (internal standard) was kindly donated by Torrent Pharmaceuticals Limited, Ahmedabad, India. HPLC grade acetonitrile, methanol and analytical grade ammonium acetate were obtained from Merck chemicals, Mumbai, India. All other chemicals used were of analytical grade.

\subsection{Preparation of liposomes}


Rivastigmine liposomal suspension was prepared by lipid layer hydration method. Briefly, solution of soya lecithin and cholesterol (4:1) solubilized in chloroform, was transferred to round bottom flask. Flask was fixed to rotary vacuum evaporator immersed in thermostat water bath set at $45^{\circ} \mathrm{C}$ and rotated till a dry lipid film was deposited on the flask walls. Aqueous phase (rivastigmine in PBS, $\mathrm{pH}=7.4$ ) was added and vortexed until the entire film deposited was consumed. The suspension was exposed to 3 freezethaw cycles, and the final preparation stored at $4^{\circ} \mathrm{C}$. These liposomes were evaluated by optical microscope as well as scanning electron microscope (SEM). Drug content analysis was carried using UV spectrophotometer. The formulation was characterized and in vitro release studies were carried [11].

\subsection{Formulation development of PLGA nanoparticles by Double emulsion (w/o/w) method}

Required amount of PLGA (50:50) was weighed and dissolved in dichloromethane (DCM). Rivastigmine solution was added drop wise into the DCM solution, vortexed and mixed to form the primary emulsion (w/o). Then the primary emulsion was added slowly into a stabilizer solution to form w/o/w emulsion containing the particles and then size reduction was carried out. Then the emulsion was kept on the magnetic stirrer at $25^{\circ} \mathrm{C}$ for overnight to evaporate the organic solvent. The data of optimization for particle size, size reduction, dispersed/continuous phase ratio is not shown in this manuscript.

\subsection{Pharmacokinetic study}

Four separate groups of rats $(\mathrm{N}=4)$, INRSM (intranasal rivastigmine solution), INRL (intranasal rivastigmine liposomes), INRNP (intranasal rivastigmine nanoparticles) and, ORSM (oral rivastigmine solution) were used for pharmacokinetic studies The overnight fasted rats were administered with different rivastigmine formulations 30 minutes following scopolamine treatment. $0.2 \mathrm{~mL}$ blood was collected from the retro orbital vein in Eppendorf tubes containing disodium EDTA (10\% w/v) at 0, 5, 10 , $15,20,30,45,60,90,120,180,240$ minutes after rivastigmine. Samples were then centrifuged at 10,000 rpm for 5 minutes and plasma was stored at $-70^{\circ} \mathrm{C}$ until analysis. The plasma samples were used to estimate rivastigmine concentrations and for AChE inhibition assay.

After 15 minute interval rats were sacrificed and brains were isolated. The whole brain was rapidly weighed and homogenised in PBS $(10 \% \mathrm{w} / \mathrm{v})$ and $500 \mu \mathrm{L}$ of this whole homogenate was taken in $1.5 \mathrm{~mL}$ centrifuge tube, centrifuged at $10,000 \mathrm{rpm}$ for 5 minutes at $-10^{\circ} \mathrm{C}$ and clear supernatant $100 \mu \mathrm{L}$ was taken for the AChE activity.

Similarly, rats treated with colchicine ICV were treated with different rivastigmine formulations and sampled at different time intervals till $2 \mathrm{hrs}$ on 12 th day post colchicine administration. On the day 24 after the induction of dementia, rat brain homogenates were prepared as described above for AchE activity.

\subsubsection{Extraction of rivastigmine}


Extraction of rivastigmine from rat plasma was carried out using liquid-liquid extraction (LLE) technique. Briefly, to an aliquot of $100 \mu \mathrm{L}$ of rat plasma, $10 \mu \mathrm{L}$ of $\mathrm{IS}(1 \mu \mathrm{g} / \mathrm{mL})$ was added and vortexed for $30 \mathrm{sec}$. Then $1 \mathrm{~mL}$ of TBME (Tertiary methyl butyl ether) was added and vortexed for 10 minutes, thereafter centrifuged at $10,000 \mathrm{rpm}$ for 5 minutes. From the supernatant $0.9 \mathrm{~mL}$ of organic solvent was aspirated and evaporated in a turbo evaporator (Zymark, Hopkinton, MA, USA) at $50 \pm 2^{\circ} \mathrm{C}$ under stream of nitrogen. The dried residue was reconstituted with $150 \mu \mathrm{L}$ of mobile phase and $50 \mu \mathrm{L}$ was injected for the HPLC analysis. A full validation according to the US-FDA guidelines was performed in rat plasma. Plasma samples were analysed by previously validated HPLC with fluorescence detection method [12].

\subsubsection{Rivastigmine quantification by HPLC [12]}

HPLC analysis was carried out on Shimadzu LC-20AD Prominence (Shimadzu Corporation, Kyoto, Japan) equipped with LC- 20AD pump, RF-10A-XL fluorescence detector, column oven (CTO-10AS VP), auto sampler SIL-20AC HT and LC solution version 1.24 SP1. The column oven temperature was maintained at $25^{\circ} \mathrm{C}$. The chromatographic separation was achieved by Interstil, ODS-3V, C18 $(250 \times 4.6 \mathrm{~mm}, 5 \mu \mathrm{m})$ column (GL Sciences Inc, Japan). Isocratic elution was performed with ammonium acetate buffer (20 $\mathrm{mM}, \mathrm{pH} 4.5)$ and acetonitrile 74:26 ( $/ \mathrm{v})$ as mobile phase. The flow rate was maintained at $1 \mathrm{~mL} / \mathrm{min}$ and injection volume was $50 \mu \mathrm{L}$. Fluorimetric detection was used and excitation and emission wavelength were of 220 and $293 \mathrm{~nm}$, respectively. The sensitivity of the detector was "Medium" and the response kept at 3. Venlafaxine (IS) was prepared separately in methanol to yield primary standard solutions with a concentration of $1 \mathrm{mg} / \mathrm{mL}$.

\subsubsection{Pharmacokinetic analysis}

The Pharmacokinetic parameters were calculated using Non-compartmental analysis, PK solution 2.1software (Summit research Services, Montrose, Colorado, USA). Area under the curve (AUC) from zero to infinity calculated using trapezoidal method. Terminal elimination rate constant $(\beta)$ obtained by plotting log linear concentration versus time, the corresponding half-life $(\mathrm{t} 1 / 2 \beta)$ was calculated by $0.693 /$ $\beta$. Plasma drug concentration of rivastigmine at time zero ( $\mathrm{CO}$ ) obtained by extrapolation of time-plasma concentration profiles. The Clearance $(\mathrm{CL})$ and volume of distribution $(\mathrm{Vd})$ were calculated as follows.

$\mathrm{CL}=$ Dose $/ \mathrm{AUC0}-\infty, \mathrm{Vd}$ (apparent $)=\mathrm{CL} / \beta$

\subsection{Pharmacodynamic studies}

The efficacy of the different formulations was assessed in acute, scopolamine induced amnesia model and in a chronic model using colchicine induced neurodegeneration.

In the acute study, following groups were used (Table 1). 
Table 1

Groups used in scopolamine induced amnesia (acute model) in rats.

\begin{tabular}{|c|c|c|c|}
\hline S.No. & $\begin{array}{l}\text { Group } \\
(n=6)\end{array}$ & Model induction & Treatment \\
\hline I & NC & Normal Saline & Normal saline \\
\hline II & SCP & \multirow{5}{*}{$\begin{array}{l}\text { Scopolamine } 1 \mathrm{mg} / \mathrm{kg} \\
\text { (i.p) } \\
\text { induced amnesia } \\
\text { (Acute model) }\end{array}$} & Normal Saline \\
\hline III & $\begin{array}{l}\mathrm{SCP}+ \\
\text { ORSM }\end{array}$ & & Rivastigmine (2.5 mg/kg, p.o.) \\
\hline IV & $\begin{array}{l}\text { SCP+ } \\
\text { INRSM }\end{array}$ & & Rivastigmine $(2.0 \mathrm{mg} / \mathrm{kg}, \mathrm{IN})$ \\
\hline V & $S C P+I N R L$ & & $\begin{array}{l}\text { Rivastigmine liposomal formulation }(2.0 \mathrm{mg} / \mathrm{kg} \text {, } \\
\text { IN) }\end{array}$ \\
\hline VI & $\begin{array}{l}\text { SCP }+ \\
\text { INRNP }\end{array}$ & & $\begin{array}{l}\text { Rivastigmine nanoparticle formulation (2.0 } \\
\mathrm{mg} / \mathrm{kg}, \mathrm{IN})\end{array}$ \\
\hline
\end{tabular}

In the chronic study, following groups were used (Table 2).

Table 2

Groups used in ICV colchicine induced dementia (chronic model) in rats.

\begin{tabular}{|c|c|c|c|}
\hline S.No. & Group $(n=8)$ & Model induction & Treatment \\
\hline I & SC & - & Normal saline \\
\hline II & ACSF & ACSF only & Normal Saline \\
\hline III & $\mathrm{COL}$ & \multirow{4}{*}{$\begin{array}{l}\text { Colchicine } \\
15 \mu g(I C V) \\
\text { induced dementia } \\
\text { (Chronic model) }\end{array}$} & Normal Saline \\
\hline IV & COL + ORSM & & Rivastigmine (2.5 mg/kg, p.o.) \\
\hline $\mathrm{V}$ & COL + INRSM & & Rivastigmine (2.0 mg/kg, IN) \\
\hline VI & $\mathrm{COL}+\mathrm{INRL}$ & & Rivastigmine liposomes $(2.0 \mathrm{mg} / \mathrm{kg}, \mathrm{IN})$ \\
\hline
\end{tabular}

Scopolamine, rivastigmine and other formulations of rivastigmine were prepared in normal saline freshly before administration. Rivastigmine solution was maintained at isotonic $\mathrm{pH}$ of $6.10 \pm 0.20$ to maintain nasal cavity pH (5.5-6.5) [13]. During the experiments, care was taken to maintain the normal functions of the nasal cavity by minimising disturbance of the mucosa with mechanical manipulation. Rats were anaesthetised with diethyl ether and rivastigmine solution was administered through the rat nasal cavity. The nasal doses were given unilaterally to the right nostril $(50 \mu \mathrm{L})$ of the rats using micro tips $(100 \mu \mathrm{L})$ attached to a micropipette. The rats were kept in the supine position for 2 minutes after administration of dose. All experiments were carried out at $20 \pm 5^{0} \mathrm{C}[14]$. 
In the acute model, the rats were trained for Morris water maze (MWM) and passive avoidance test (PAT) tasks in the first 4 days. On 5th day, 30 min after scopolamine administration, respective treatments were given to all the groups. 15 min thereafter the test trials were conducted in MWM and PAT.

In the chronic model, the rats were trained for MWM and PAT tasks for first four days followed by intracerebroventricular (i.c.v.) administration of colchicine on day 6 . After establishing the dementia on day 10 of the colchicine administration, the drug treatment was initiated from day 11 to 24 . The effects of different drug treatments on dementia were assessed on day 14 and 21 . On these particular days the swim test was performed 15 min after the administration of different treatments and escape latencies, residence time and peripheral swim time were recorded [15]. The retention trials for PAT on day 17 and 23 were performed on days 17 and 23, 15 minutes after the administration of the drug. Following rivastigmine administration, 15 minutes lag time for assessment was chosen based on pilot PK and PD studies where Cmax was observed.

\subsection{ICV Colchicine induced dementia}

On 6th day, surgery was performed as described [16]. Rat was anesthetized with sodium pentobarbital (45 mg/kg, i.p.) and positioned in stereotaxic apparatus. The head was positioned in a frame and a midline sagittal incision was made in the scalp. Two holes were drilled through the skull at coordinates ( $0.8 \mathrm{~mm}$ posterior to bregma, $1.8 \mathrm{~mm}$ lateral to sagittal suture and $3.6 \mathrm{~mm}$ beneath the cortical surface) to place the injection canula. Rats were intracerebroventricularly infused with either artificial cerebrospinal fluid (ACSF; in mM: $147 \mathrm{NaCl}, 2.9 \mathrm{KCl}, 1.6 \mathrm{MgCl}_{2}, 1.7 \mathrm{CaCl}_{2}$ and 2.2 dextrose) or $15 \mu \mathrm{g}$ of colchicine in $5 \mu \mathrm{l}$ ACSF using a Hamilton microsyringe positioned in the injection cannula. To promote back diffusion, the microsyringe was left in place for a period of $2 \mathrm{~min}$ following injection. The scalp was then closed with suture. After surgery, all animals received gentamicin $(5 \mathrm{mg} / \mathrm{kg}$, i.p.) to prevent sepsis. Special care was taken during the postoperative period to provide food and water inside the cage of the rat. In sham-operated rats, surgery was identical except for injection of the solution.

\subsection{Behavioral Assessment}

\subsubsection{Locomotor activity [15]}

Locomotor activity was assessed in animals using digital photoactometer (INCO, India) which contains a cage that is $30 \mathrm{~cm}$ long and $30 \mathrm{~cm}$ deep with a wire mesh at the bottom. The apparatus was placed in a dark, light and sound attenuated testing room. Before locomotor task, animals were placed individually in the activity meter for $3 \mathrm{~min}$ for habituation. Then, the ambulatory movements were recorded for a period of $5 \mathrm{~min}$ and expressed in terms of total photo beam counts for 5 min per animal.

\subsubsection{Assessment of cognitive performance:}

Spatial learning and memory were assessed in Morris water maze. Learning and memory were assessed in passive avoidance.

\section{A. Morris water maze test}


Modified Morris water maze test was used [17]. The pool was positioned in the middle of a dimly lit testing room with distant visual clues which could be used by rats for spatial orientation. Maze consisted of a circular water pool $(140 \times 45 \mathrm{~cm})$ divided into four equally sized quadrants (NE, SE, SW, and NW) by two imaginary diagonal lines running across the centre of the pool. The pool was filled with water maintained at temperature of $23 \pm 2^{\circ} \mathrm{C}$. In NE quadrant (target quadrant) equidistant from the centre and edge of the pool, a platform of $25 \mathrm{~cm}$ height and $10 \mathrm{~cm}$ diameter was invisibly placed submerged $1 \mathrm{~cm}$ below water surface. Rats were gently placed in each quadrant facing the wall and trained to locate the hidden black platform and trial was terminated when rat reached the platform or after 60 seconds. Accordingly, rats were subjected to one session of four trials per day for four consecutive days. All rats were left on platform for 30 seconds and then removed and towel dried. Inter trial interval was kept a constant 180 seconds throughout the study. In the probe trial, platform was removed and rats allowed to swim for a 60 seconds trial period. Data was collected in the form of escape latencies to find the platform; percent of time spent over target quadrant.

\section{B. Passive avoidance test}

The passive avoidance apparatus consists of larger illuminated compartment and smaller dark compartment. Each consists of a square box, with a floor grid of $50 \times 50 \mathrm{~cm}$ and wooden walls of $35 \mathrm{~cm}$ height in larger compartment. Larger box was illuminated by 15 watts Philips CFL lamp. A smaller dark compartment $15 \times 15 \mathrm{~cm}$ with electrified floor connected to a constant current stimulator. An opening $6 \times 6$ $\mathrm{cm}$ in between the two compartments which was closed using a transparent plexi glass sliding door. Test procedure, was carried out in three phases, exploration, learning and retention [18].

Firs day, the animal was placed in the centre of the illuminated larger compartment facing away from the entrance to the small darker compartment and allowed to explore the apparatus i.e. both compartments for 3 minutes. Total time spent in the larger and smaller compartments and number of crossings (from larger to smaller compartments and vice versa; a measure of exploratory behaviour) were noted. On the second day, animal was placed in illuminated larger compartment facing away from the entrance to darker compartment. Once the animal entered the darker compartment, the entrance to larger compartment was closed and three strong electric foot shocks ( $50 \mathrm{~Hz}, 0.5 \mathrm{~mA}, 2 \mathrm{sec}$ ) with ten second intervals were applied. On the third day, rats were placed in the illuminated larger compartment and allowed to explore both the compartments for 3 minutes. The parameters assessed are latency time to enter into darker compartment, time spent in the darker and brighter compartments and number of crossings between the compartments.

\subsection{AChE assay}

AChE activity and its inhibition by rivastigmine in rat plasma and whole brain homogenate were estimated by ex vivo method using Ellman's method [19]. In this assay, thiol ester acetylthiocholine was used instead of the oxyesteracetylcholine, as suggested by Ellman. Thiocholine, which was produced due to the hydrolysis of acetylthiocholine (ACTI) by AChE, was estimated by making it to react with $5,5^{\prime}$ dithiobis-(2-nitrobenzoic acid) (DTNB). The chemical, 5-thio-2-nitrobenzoic acid (yellow colour complex), 
which was released due to reaction was estimated using UV spectrophotometer in the range of 400 to $420 \mathrm{~nm}$ with maximal absorption at $412 \mathrm{~nm}$. $100 \mu \mathrm{L}$ of blank rat plasma was taken in the polypropylene vials. To this $2.8 \mathrm{~mL}$ of phosphate buffer $(0.01 \mathrm{M}, \mathrm{pH} 8)$ and $100 \mu \mathrm{L}$ of DTNB reagents were added and mixed well. Then $20 \mu \mathrm{L}$ of ACTI $(26.01 \mathrm{mg} / \mathrm{ml}$ of milli q water) was added to the contents of the cuvette to initiate the enzyme reaction. The reaction kinetics was monitored at different time intervals $(0,1,2,3,4$ minutes) and absorbance was noted. $2.8 \mathrm{~mL}$ buffer $(0.01 \mathrm{M}, \mathrm{pH} 8.0)$ and $100 \mu \mathrm{L}$ DTNB reagent served as blank. Concentrations in the range of $0.03-30 \mathrm{mM}$ were prepared using methanol as a diluent. Previously AChE activity of the blank plasma sample without adding rivastigmine was measured and that response was taken as a control. Substrate concentration and reaction time were optimised such that sufficient concentration of ACTI is available to bind with enzyme to initiate the reaction and also it should not saturate the enzyme. In this study varying concentrations $(100-1000 \mu \mathrm{M})$ of ACTI were prepared and reaction kinetics were observed. In general, the maximum time should be either 2-4 minutes or even less, because enzyme might undergo degradation. In the present study up to 4 minutes reaction kinetics (changing the optical density or absorbance) was measured. One unit of AChE activity is defined as number of $\mu \mathrm{mol}$ of acetylthiocholine iodide hydrolyzed per minute. The specific activity of AChE is expressed in moles/min/mg of protein [20].

AChE Activity $=$ (abs. per $\mathrm{min} \times 1000 \times$ volume of enzyme $) /(13600 \times$ TRM $)$
AChE Activity $=$ above value $\times 1000($ nano $\mathrm{molar} / \mathrm{min} / \mathrm{mL})$

Where, $13600=$ molar extinction coefficient of DTNB: 1000- molar conversion factor: (TRM) total reaction mixture $3 \mathrm{~mL}$ : volume of enzyme $(0.1 \mathrm{~mL})$.

\subsection{PK-PD Study}

PK and PD studies were carried in scopolamine induced amnesia rats.

\section{A. PD analysis}

PD (AChE) parameters were calculated using WinNonlin 5.2 (Pharsight Corporation, USA) software. Various PD models (Simple Emax, Inhibitory Emax and Sigmoid Emax) were attempted using the software. The PD models were optimized based on the correlation (observed, predicted values), weighted correlation, Akaike Information Criterion (AIC) and Schwarz Bayesian Criterion (SBC). A measure of goodness of fit was arrived based on maximum likelihood, on the comparison of several models for a given data set, and the model associated with the smallest AIC. The best PD model was selected that had smaller AIC, BIC values and good correlation (>0.99) function.

\section{B. PK-PD modelling}

PK-PD model was developed to describe the effect of plasma concentration of rivastigmine on the AChE inhibition following intranasal administration. A total of nine PK models were developed to fit the pharmacokinetic profiles of rivastigmine. The best PK model was chosen based on the PK parameters 
output results standard error, \% CV, residual values, correlation coefficient (observed vs. predicted), weighted correlation, AIC and SBC values. Five PD models were developed and checked their fitting performance in terms of standard error, \% CV, residuals and diagnostic plots.

\subsection{Data analysis}

Data was statistically analysed using Graph Pad Prism 5.0 software. Mean values were analysed by oneway ANOVA followed by Tukey post-hoc test. All data were expressed as the mean $\pm S E$. $p<0.05$ was considered statistically significant at $95 \%$ confidence interval.

\section{Results}

\subsection{Pharmacokinetic profiles of different formulation in scopolamine induced amnesic rats}

Nasally administered rivastigmine and its formulations (INRSM, INRL, INRNP) showed rapid absorption through nasal mucosa as demonstrated by high plasma drug concentrations at 5 minutes. The plasma drug levels declined rapidly in nasal rivastigmine nanoparticles (INRNP) and oral pure drug (ORSM) whereas maintained in INRSM and INRL (Fig. 2). The systemic availability (AUC) and peak plasma concentration were increased in the order of liposomes $>$ nasal pure drug and PLGA nanoparticles. PLGA nanoparticles showed significantly lower systemic bioavailability and larger volume of distribution when compared to liposomes as well as nasal pure drug. Rivastigmine loaded liposomes had a lower clearance rate compared to nanoparticles as well as nasal pure drug. The absolute bioavailability of rivastigmine was significantly higher for liposomes compared to pure drug and nanoparticles. Orally administered rivastigmine had the least Cmax, bioavailability (AUC) and maximum clearance where only a fraction of drug reached the systemic circulation. It had shown a larger volume of distribution $(29.23 \pm 14.35 \mathrm{~L} / \mathrm{kg})$ and extremely higher clearance rate $(1.50 \pm 0.76 \mathrm{~L} / \mathrm{min})$ resulting in faster elimination from the plasma (fig. 3).

\subsection{Effect of different rivastigmine formulations on scopolamine induced memory deficit}

\section{A. Locomotor activity}

Locomotor activity as assessed by actophotometer was not significantly different among the groups as shown in fig 4.

\section{B. Morris water maze}

Time taken to find the platform was significantly higher in scopolamine induced amnesic rats compared to control rats indicating the memory deficit in these rats (Fig. 5). Meanwhile, all rivastigmine treated groups exhibited significantly shortened escape latency times, but the extent of reduction was highly significant in INRL group compared to scopolamine alone treated group. The INRL group restored the memory almost comparable to normal control. 
Scopolamine alone treated rats spent less time in target quadrant and more time in periphery compared to control as they failed to remember the platform location, indicating the loss of retentive memory in these rats. On the other hand, all the rivastigmine treated groups spent significantly more time in the target quadrant and less time in periphery indicating the restoration of retention memory in these rats. This beneficial effect was in the order INRL $>$ INRSM $>0 R S M>I N R N P$ (Fig. 5). Rivastigmine as a liposomal nasal formulation had shown better performance in working and reference memory in Morris water maze when compared to the other treatments in scopolamine induced amnesia.

\section{Passive avoidance test}

In the acquisition test trial, no significant difference was found among all the groups. Scopolamine alone treated rats have exhibited lower latency times i.e., time taken to enter the darker compartment and spent higher time in darker chamber compared to control, as they fail to remember the electric shock in the darker compartment. Only the rivastigmine loaded liposomal formulation given through intranasal route has significantly reversed the effect of scopolamine, as evident by the enhanced latency times to enter the darker chamber and reduced residence time in darker chamber as compared to scopolamine alone treated as well as ORSM groups. Whereas, all other treatment groups were not significant (fig 6A). There was no significant difference among the groups in the number of crossings between the darker and lighter compartments (fig 6B).

\subsection{Effect of different rivastigmine formulations on acetylcholinesterase activity in plasma and whole brain homogenate in rats (Ex vivo experiment)}

On comparison of all treatments, the peak AChE inhibition was noted at $15 \mathrm{~min}$ time interval by INRL (81.92 \%), followed by INRSM (71.95\%) at 10min, then ORSM (71.11\%) at $60 \mathrm{~min}$ and INRNP $(59.86 \%)$ at $45 \mathrm{~min}$ in the plasma sample (fig 7a). At $15 \mathrm{~min}$, INRL showed maximal AChE inhibition compared to all the treatment groups in plasma (fig $7 b$ ).

In case of whole brain homogenate, AChE inhibition was estimated only at $15 \mathrm{~min}$ interval. The peak AChE inhibition was noted by INRL (36.26\%), followed by INRSM (33.34\%), then INRNP (10.97\%) at last tailed by ORSM $(6.16 \%)$ as shown in figure $7 \mathrm{c}$.

\subsection{Effect of different rivastigmine formulations on colchicine induced memory deficit}

\section{A. Locomotor activity}

The spontaneous locomotor activity did not differ significantly between the groups as shown in fig 8 .

\section{B. Morris water maze}

On the probe day, there was no significant difference in escape latency and residence times between the treatment groups. On day 14 ( $1^{\text {st }}$ retention test) and day 21 ( $2^{\text {nd }}$ retention test), COL group displayed significantly increased escape latency and reduced time spent in the target quadrant over the SC and 
ACSF groups indicating the memory deficit induced in the colchicine alone treated rats. Meanwhile, all the treatment groups exhibited significantly reduced escape latency times, and increased time spent in the target quadrant over COL rats on $21^{\text {st }}$ day and INRL was found to be significant over ORSM and INRSM groups in residence time (fig. 9).

\section{Passive avoidance test}

No significant difference was found between the groups in all the parameters, latency to enter darker chamber, time spent in darker chamber and number of crossings assessed in $1^{\text {st }}$ retention test, which was performed before colchicine administration (fig. 10). In the $2^{\text {nd }}$ and $3^{\text {rd }}$ retention test assessed on $17^{\text {th }}$ and $23^{\text {rd }}$ day post colchicine administration, $\mathrm{COL}$ showed significantly higher number of crossings and increase in time spent in dark chamber compared to sham and ACSF indicating the memory deficit induced by colchicine, as it failed to remember the electric shock. Meanwhile, all the treatment groups exhibited reduced number of crossings as compared to $\mathrm{COL}$ in $2^{\text {nd }}$ retention test. In $3^{\text {rd }}$ retention test, INRL and INRSM significantly decreased time spent in dark chamber and number of crossings compared to $\mathrm{COL}$ (fig. 10).

\subsection{Effect of rivastigmine formulations on acetylcholinesterase activity in plasma and whole brain homogenate of rats in ICV colchicine induced rats (Ex vivo experiment).}

Rivastigmine along with its formulations were tested for their effect on acetylcholinesterase inhibition in colchicine induced dementia. On comparison of all treatments in rat plasma, the peak AChE inhibition was noted at $5 \mathrm{~min}$ time interval by INRL $(89.86 \%)$, followed by INRSM $(76.75 \%)$ at $15 \mathrm{~min}$ and ORSM $(69.56 \%)$ at $45 \mathrm{~min}$ (fig 11A). At 15min, AChE inhibition was significantly higher in INRL group compared to COL, ORSM and INRSM plasma samples (Fig. 11B). The treatments with whole brain homogenate were estimated at $15 \mathrm{~min}$ interval only and the peak AChE inhibition was noted by INRL (45.38 \%), followed by INRSM $(36.14 \%)$ and then by ORSM $(24.09 \%)$ as shown in figure 11 C.

\subsection{Pharmacokinetic and pharmacodynamic modelling in scopolamine induced amnesia}

\section{PD model}

The best PD model selected was Simple Emax ((Effect $\mathrm{C}=0$ at E0; Effect at $\mathrm{C}=$ infinity). This model was used to estimate the PD parameters namely Emax, EC50, E0 and $\mathrm{AUE}_{0-\mathrm{t}}$ for plasma AChE ex vivo inhibition studies in rats. The PD effect of rivastigmine (\% AChE inhibition) was in close agreement with the concentration-time course (i.e. PK profile) in plasma. Intranasally administered liposomes had significantly higher Emax (maximum inhibition $81.92 \%$ ) than nanoparticles $(59.86 \%$ ) as well as pure drug (71.95\%). Orally administered rivastigmine showed maximum AChE inhibition $(71.11 \%)$ at 60 minutes (fig. 7A).

\section{PK model}


Developed PK model was perfectly fitted into two compartment models. Among the PK models developed, two-compartment, intravenous bolus, no lag time, micro constants and first order elimination seems to be the best fitted PK model to describe the PK parameters of rivastigmine loaded liposomes. The developed model showed smaller \% CV, AIC, SBC values and observed-predicted concentrations were very close (smaller residuals) and correlation was more than 0.99 . All these parameters indicate that rivastigmine PK parameters could be best fitted into two compartment model with first order elimination as shown below equation.

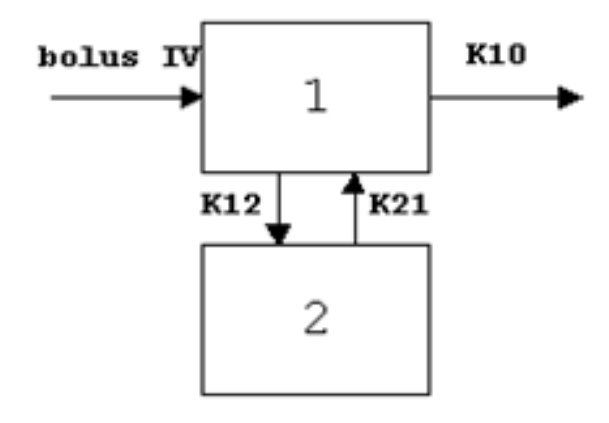

$\mathrm{C}(\mathrm{T})=\mathrm{A} \times \exp (-\mathrm{Alpha} \times \mathrm{T})+\mathrm{B} \times \exp (-\mathrm{Beta} \times \mathrm{T}) ; \mathrm{K} 10=\mathrm{CL} / \mathrm{V} 1 ; \mathrm{K} 12=\mathrm{CLD} 2 / \mathrm{V} 1 ; \mathrm{K} 21=\mathrm{CLD} 2 / \mathrm{V} 2$

The inhibition of AChE in plasma could be best explained by simple Emax model with Effect $\mathrm{C}=0$ at 0 , $\mathrm{C}=$ infinity at Emax described by equation as shown in below equation.

$\mathrm{E}=\mathrm{Emax} \times \mathrm{Ce}) /(\mathrm{Ce}+\mathrm{ECe} 50)$

The PK-PD results of liposomes are illustrated in table 3. The developed PK model was found to best describe the disposition kinetics of rivastigmine loaded liposomes following intranasal administration (fig. 12A). PD data of rivastigmine in rat plasma was best fitted into the simple Emax model (fig. 12B). Results revealed the reduction in AChE inhibition with time as the plasma concentrations decrease indicating the direct relationship between the PK and PD (fig. 12C). The PK and PD of rivastigmine in plasma could be well described by these models that assumes that no time delay between the activity and concentrations in plasma. Histersis curve indicates drug concentration in plasma (Cp) and drug concentration in the effective site (Ce) are correlating as shown in figure 12D.

The PK-PD link model showed that smaller standard error for KEO (rate of drug loss from the effective compartment) and other PD parameters. The predicted AChE inhibition or plasma concentration and experimental AChE inhibition or plasma concentrations are fairly matching. This result indicates that PKPD link model explains the time vs AChE inhibition very well.

Table 3 Summary of estimated PK-PD parameters of rivastigmine loaded liposomes in rat plasma 


\begin{tabular}{|llll|}
\hline Parameter & Units & Std error & CV\% \\
\hline Emax & $\%$ & 71.4033 & 3.46 \\
\hline ECe50 & $\mathrm{ng} / \mathrm{mL}$ & 0.0000001 & 61878.96 \\
\hline KEO & & 253162.7668 & 25778658.00 \\
\hline
\end{tabular}

\section{Discussion}

Cholinesterase inhibitors are best-established class of therapeutics for treatment of dementia of Alzheimer's type (DAT). Cholinesterase inhibitors enhance the availability of acetylcholine (ACh) at the synapse by inhibiting acetylcholine esterase enzyme inhibition (AChE inhibition). Acute and chronic treatment with cholinesterase inhibitors has been shown to improve cognitive function in animal models and in patients with DAT [5]. Rivastigmine, a reversible dual cholinesterase inhibitor is more tolerable and widely used choice of drug among the available drugs. However, rivastigmine being hydrophilic and undergoing first pass metabolism, exhibits low CNS bioavailability [21]. Nanoformulations including liposomes and PLGA nanoparticles can encapsulate hydrophilic drugs and deliver efficiently nose to brain by improving their penetration, escaping the mucociliary clearance and facilitating their transport across the mucosal barrier [9].

In the present study, intranasally administered rivastigmine liposomes had significantly improved pharmacokinetic parameters compared to the nasal pure rivastigmine, nasal rivastigmine nanoparticles and oral rivastigmine. Liposomes produced rapid initial increase in plasma drug levels presumably due to the release of adsorped free drug present on the liposomal surface [22]. Moreover, liposomes had maintained the concentration up to 3-4 $\mathrm{h}$ and prevented the sudden peak to trough levels as in ORSM group (Fig. 2). An ideal drug delivery system should have properties such as high Cmax, low clearance rate, and enhanced AUC, MRT and half-life. The developed rivastigmine liposomal formulation showed such increase in the Cmax and AUC (Fig. 3) indicating that it could reduce the toxic effects. These results are in accordance with the previous reports on the liposomal formulation for other drugs [23]. Smaller volume of distribution and lower clearance rate was found for liposomes. This is possibly due to smaller particle size, hydrophilic nature, sterically stabilized liposomes which could have minimized the opsonization and reduced the RES uptake leading to longer circulation time and higher MRT [24]. Liposomal formulation factors such as particle size, surface charge, chitosan coating and PEGylation play a major role in the pharmacokinetic parameters of rivastigmine. On the other hand, rivastigmine loaded PLGA nanoparticles showed significantly higher clearance rate, subsequently leading to shorter half-life and less systemic exposure. The other possible reason could be due to the higher uptake by RES/macrophages and hydrophilic surface of nanoparticles [25].

The cholinergic system in basal forebrain plays an important role in learning and memory. Scopolamine interferes with memory and cognitive function by blocking $M_{1}$ type muscarinic receptors in the brain regions [26]. The present study compared the effects of cholinesterase inhibitor, rivastigmine between 
pure form and the novel formulations administered via intranasal route in the scopolamine induced memory impaired rats in the MWM and PAT tasks.

There was no significant difference among the treatment groups in locomotor activity assessed with actophotometer indicating no effect of treatment on the motor activity of the animals [15]. Rivastigmine at a dose of $2.5 \mathrm{mg} / \mathrm{kg}$ via oral route, inhibited the cholinesterase enzyme in the cortex and hippocampus by $20-30 \%$, and significantly reduced the effects of scopolamine on reference and working memory in MWM and PAT [27]. INRL treatment significantly reversed the memory deficit induced by scopolamine superior to other formulations as assessed in MWM and PAT tasks. Further, this was supported by pharmacokinetic parameters as well as acetylcholinesterase inhibition studies. The acetylcholinesterase inhibition was maximal for INRL in both rat plasma and whole brain homogenate and the order of inhibition were INRL > INRSM > ORSM and INRNP. Thus, the intranasal liposomal rivastigmine (INRL) treatment has beneficial effect over the amnesia induced by scopolamine superior to the conventional oral treatment and also intranasal pure rivastigmine administration.

In chronic model, colchicine binds irreversibly to tubulin to form tubulin dimers and prevents addition of tubulin molecules to the fastgrowing end, there by inhibiting microtubule assembly and disrupting microtubule polymerization resulting in cell death and subsequently cognitive impairment [28]. In the present study, colchicine caused significant loss of memory as evident in MWM and PAT. It has been reported that colchicine produces a time and dose dependent changes (anatomical, behavioural and neurochemical) at maximum of 14-21 days following the ICV colchicine administration [29]. As observed with scopolamine model, the INRL group significantly rescued the memory deficit induced by colchicine administration.

Thus, the present study demonstrated that rivastigmine along with its formulations were effective in enhancing the memory deficits in both the models employed. INRL was found to be more effective than the other treatments in all the parameters assessed in both the models. The above statement was supported by acetylcholinesterase enzyme inhibition in plasma and whole brain homogenates. The \% inhibition was higher in the order of INRL > INRSM and ORSM. Here the INRNP group i.e. nanoparticle formulation was excluded from the chronic model as it showed very poor results in the acute model (scopolamine induced amnesia).

\section{Conclusion}

Based on the results, nasal delivery of rivastigmine liposomes showed ideal pharmacokinetic characteristics with rapid absorption, enhanced systemic bioavailability, half-life and mean residence time superior to other formulations. Besides, it reversed the memory deficit induced by acute (scopolamine) as well as chronic (colchicine) models and exhibited a good agreement between pharmacokinetic and pharmacodynamic activities. Therefore, nasal route of rivastigmine liposomal formulation can be an ideal choice for treating dementia related to Alzheimer's disease. 


\section{Abbreviations}

Pharmacokinetic and pharmcodynamic (PK-PD) modelling, Poly D,L-lactic-co-glycolic acid (PLGA), Morris water maze (MWM)

\section{Declarations}

Ethical standards: The animal experimentation protocol was approved by the Institutional Animal ethical committee of Manipal University

Conflict of interest: The authors declare that they have no conflict of interest.

\section{Author contributions}

Conceptualization: SKR and CMR; Methodology: SKR and KA; Validation: SKR and NK; Formulation development: KA; In-vivo experimentation: SKR and CMR; Data analysis and initial draft preparation: SLB, NK and SKR; All authors agree to be accountable for all aspects of work ensuring integrity and accuracy.

Availability of data: Data is included in the form of excel file.

\section{References}

1. GBD 2016 Neurology Collaborators (2019) Global, regional, and national burden of neurological disorders, 1990-2016: a systematic analysis for the Global Burden of Disease Study. Lancet Neurol 18:459-480. https://doi.org/10.1016/S1474-4422(18)30499-X

2. Apostolova LG (2016) Alzheimer Disease. Continuum (Minneapolis, Minn.), 22:419-434. https://doi.org/10.1212/CON.0000000000000307

3. Alzheimer's Association (2018) Alzheimer's disease facts and figures. Alzheimers Dement 14:367429.

4. DeTure MA, Dickson DW (2019) The neuropathological diagnosis of Alzheimer's disease. Mol Neurodegeneration 14:32. https://doi.org/10.1186/s13024-019-0333-5

5. Sharma K (2019) Cholinesterase inhibitors as Alzheimer's therapeutics. Mol Med Rep 20:1479-1487. https://doi.org/10.3892/mmr.2019.10374

6. Polinsky RJ (1998) Clinical pharmacology of rivastigmine: a new-generation acetylcholinesterase inhibitor for the treatment of Alzheimer's disease. Clin ther 20:634-647. https://doi.org/10.1016/s0149-2918(98)80127-6

7. Dhillon S (2011) Rivastigmine Transdermal Patch. Drugs 71:1209-1231. https://doi.org/10.2165/11206380-000000000-00000

8. Agrawal M, Saraf S, Antimisiaris SG, et al (2018) Nose-to-brain drug delivery: An update on clinical challenges and progress towards approval of anti-Alzheimer drugs. J Contro Release 281:139-177. https://doi.org/10.1016/j.jconrel.2018.05.011 
9. Vieira DB, Gamarra LF (2016) Getting into the brain: liposome-based strategies for effective drug delivery across the blood-brain barrier. Int J Nanomedicine 11:5381-5414.

https://doi.org/10.2147/IJN.S117210

10. Arumugam K, Chamallamudi MR, Mallayasamy SR et al (2009) Gender differences in the pharmacokinetics of rivastigmine in rats. Arzneimittelforschung 59:493-497.

11. Arumugam K, Subramanian GS, Mallayasamy SR et al (2008) A study of rivastigmine liposomes for delivery into the brain through intranasal route. Acta pharmaceutica (Zagreb, Croatia) 58:287-297. https://doi.org/10.2478/v10007-008-0014-3

12. Arumugam K, Chamallamudi MR, Gilibili RR, et al (2011) Development and validation of a HPLC method for quantification of rivastigmine in rat urine and identification of a novel metabolite in urine by LC-MS/MS. Biomed Chromatogr 25:353-361. doi:10.1002/bmc.1455

13. Chonkar A, Nayak U, Udupa N (2015) Smart Polymers in Nasal Drug Delivery. Indian J Pharm Sci 77:367-375. https://doi.org/10.4103/0250-474x.164770

14. Krishnan J, Arun P, Chembukave B, et al (2017) Effect of administration method, animal weight and age on the intranasal delivery of drugs to the brain. J Neurosci Methods 286:16-21. https://doi.org/10.1016/j.jneumeth.2017.05.012

15. Garg R, Kumar A (2008) Possible role of citalopram and desipramine against sleep deprivationinduced anxiety like-behavior alterations and oxidative damage in mice. Indian J Exp Biol 46:770776.

16. Bokare AM, Bhonde M, Goel R, Nayak Y (2018) 5-HT6 receptor agonist and antagonist modulates ICV-STZ-induced memory impairment in rats. Psychopharmacology (Berl). 235:1557-1570. doi: 10.1007/s00213-018-4866-Z

17. Nampoothiri M, Kumar N, Venkata Ramalingayya G, et al (2017) Effect of insulin on spatial memory in aluminum chloride-induced dementia in rats. Neuroreport 28:540-544. https://doi.org/10.1097/WNR.0000000000000799.

18. Kumar SEP, Bairy KL, Nayak V, et al (2019) Amelioration of Aluminium Chloride (AICl3) Induced Neurotoxicity by Combination of Rivastigmine and Memantine with Artesunate in Albino Wistar Rats. Biomed Pharmacol J 12(2).

19. Pohanka M (2015) Determination of acetylcholinesterase and butyrylcholinesterase activity without dilution of biological samples. Chem Pap 69:1044-1049. https://doi.org/10.1515/chempap-20150117

20. Fernandes J, Mudgal J, Rao, CM, et al (2018) N-acetyl-L-tryptophan, a substance-P receptor antagonist attenuates aluminum-induced spatial memory deficit in rats. Toxicol Mech Methods 28:328-334. https://doi.org/10.1080/15376516.2017.1411412

21. Dighe SN, De la Mora E, Chan S, et al (2019) Rivastigmine and metabolite analogues with putative Alzheimer's disease-modifying properties in a Caenorhabditis elegans model. Commun Chem 2:35. https://doi.org/10.1038/s42004-019-0133-4 
22. Puri A, Loomis K, Smith B (2009) Lipid-based nanoparticles as pharmaceutical drug carriers: from concepts to clinic. Crit Rev Ther Drug 26:523-580. doi: 10.1615/critrevtherdrugcarriersyst.v26.i6.10.

23. Hong SS, Oh KT, Choi HG, Lim SJ (2019). Liposomal Formulations for Nose-to-Brain Delivery: Recent Advances and Future Perspectives. Pharmaceutics 11:540. https://doi.org/10.3390/pharmaceutics 11100540

24. Sercombe L, Veerati T, Moheimani F, et al (2015) Advances and Challenges of Liposome Assisted Drug Delivery. Front Pharmacol 6:286. https://doi.org/10.3389/fphar.2015.00286

25. Behzadi S, Serpooshan V, Tao W (2017) Cellular uptake of nanoparticles: journey inside the cell. Chem Soc Rev 46:4218-4244. https://doi.org/10.1039/c6cs00636a

26. Svoboda J, Popelikova A, Stuchlik A (2017) Drugs Interfering with Muscarinic Acetylcholine Receptors and Their Effects on Place Navigation. Front Psychiatry 8:215. https://doi.org/10.3389/fpsyt.2017.00215

27. Bejar C, Wang RH, Weinstock M (1999) Effect of rivastigmine on scopolamine induced memory impairment in rats. Eur J Pharmacol 383.231-240.

28. Nakayama T, Sawada T (2002) Involvement of microtubule integrity in memory impairment caused by colchicines. Pharmacol Biochem Behav 71:119-138.

29. Emerich DF, Walsh TJ (1990) Cholinergic loss and cognitive impairment following intraventricular injections of colchicines. Brain Res 517:57-167.

\section{Figures}




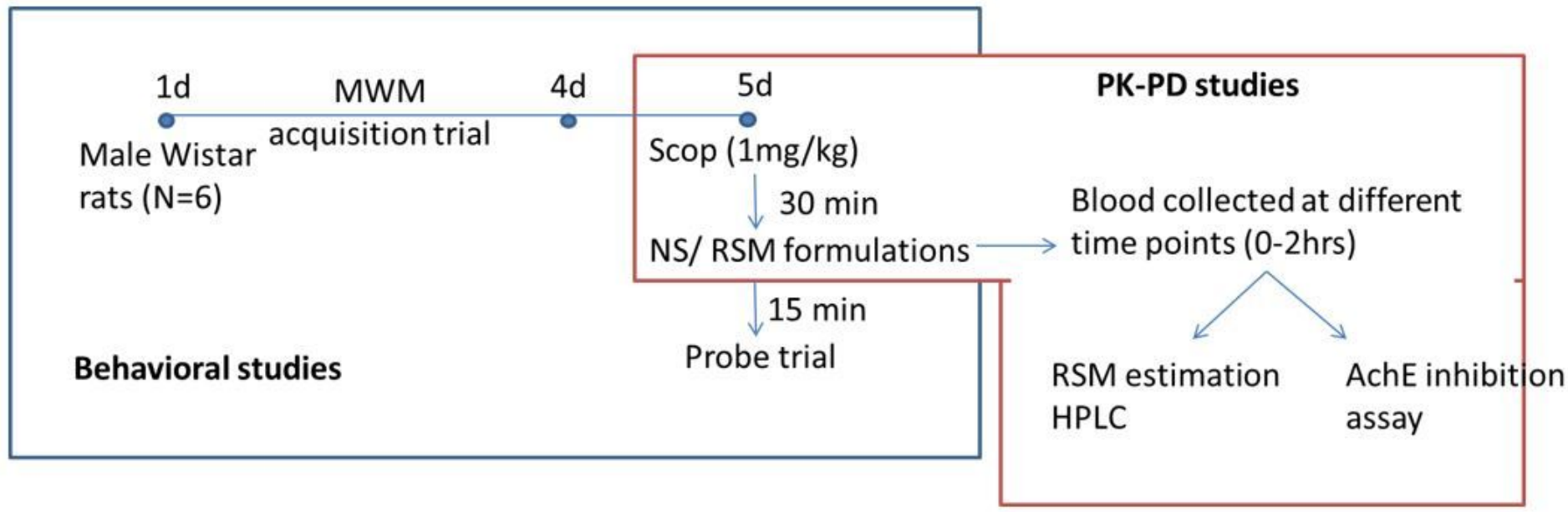

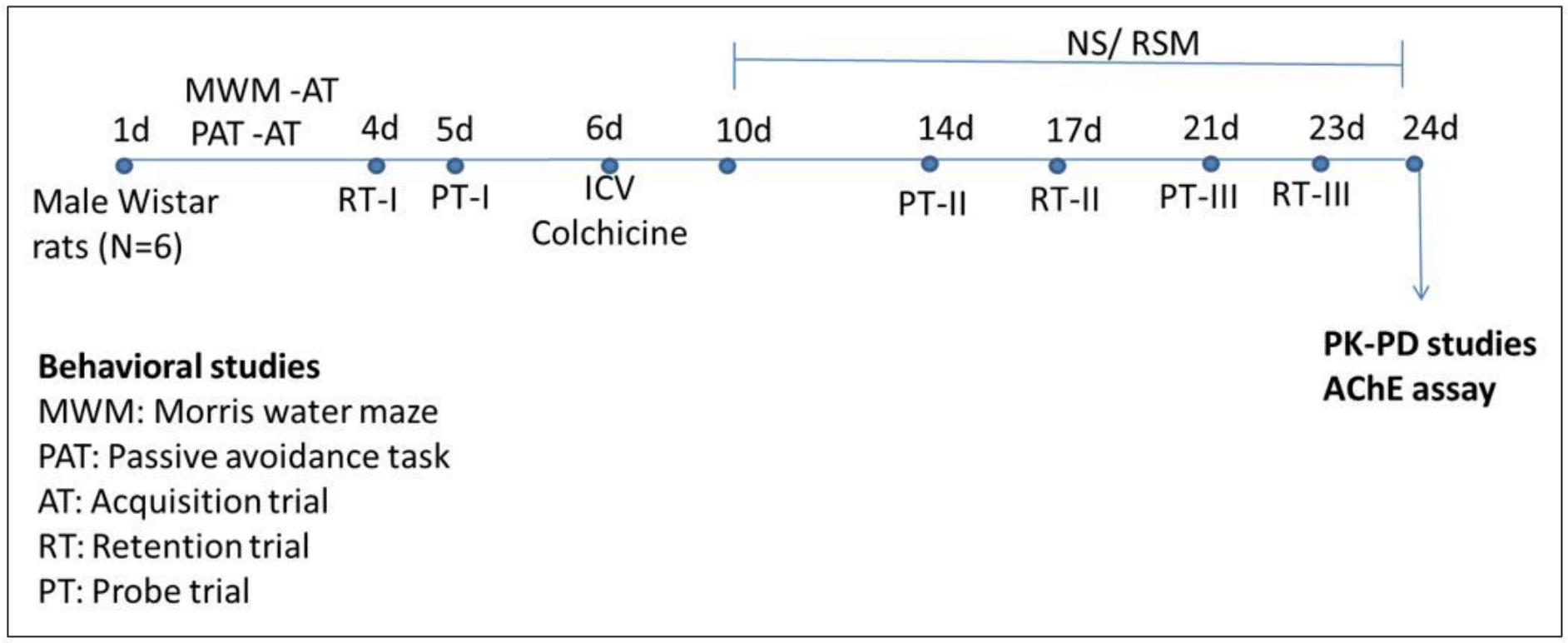

Figure 1

Experimental protocol for acute and chronic models of dementia followed in the study 


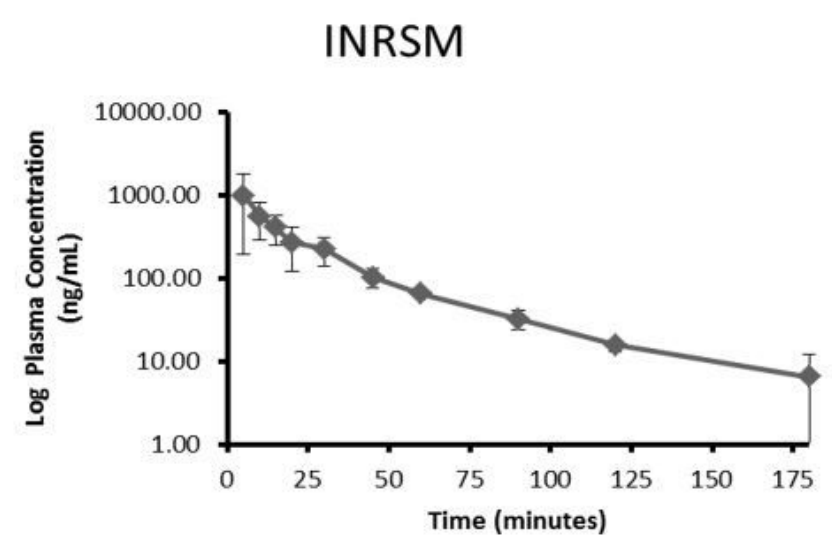

INRNP

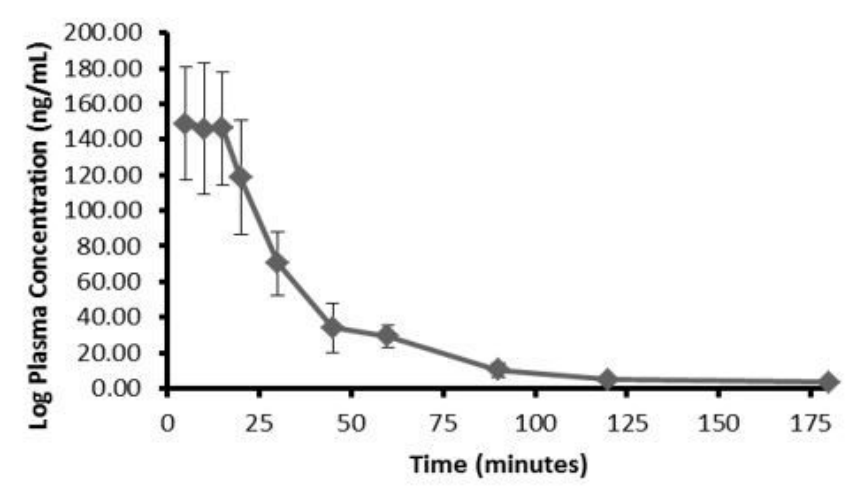

INRL

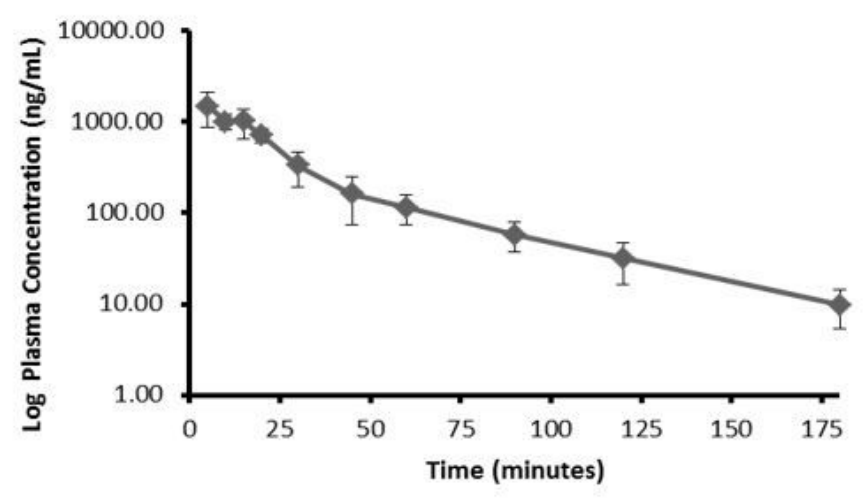

ORSM

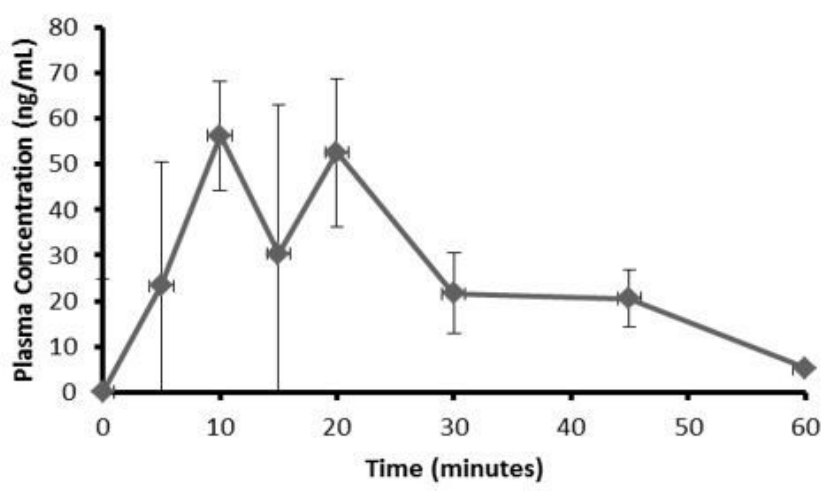

\section{Figure 2}

Plasma concentration-time curve of different formulation administered through intranasal or oral route in scopolamine induced amnesia rats $(n=4)$. 

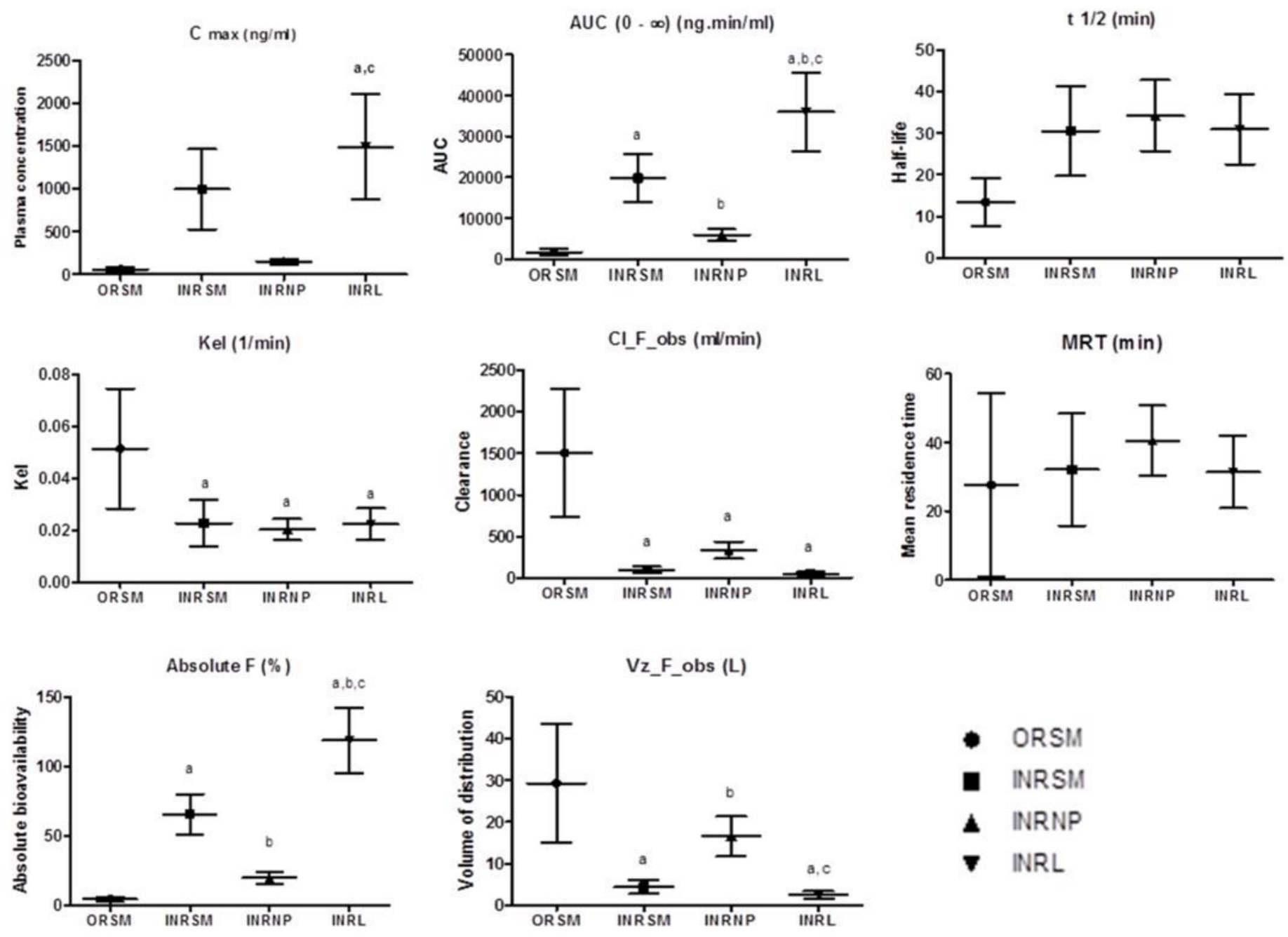

Figure 3

Pharmacokinetic features of different rivastigmine formulations. Maximum concentration (Cmax), Area under the curve (AUC), Elimination rate constant (Kel), Half-life ( $t$ 1 $1 / 2)$, Clearance (Cl_F_obs), Mean residence time (MRT), Absolute bioavailability (\%F), Volume of distribution (Vz_F_obs) of rivastigmine and its formulations in scopolamine induced rats. Data represented as mean $\pm S D$, ANOVA followed by post-hoc test $* a=p<0.05$ vs. ORSM; ${ }^{*} b=p<0.05$ vs. INRSM; ${ }^{*} c=p<0.05$ vs. INRNP). 


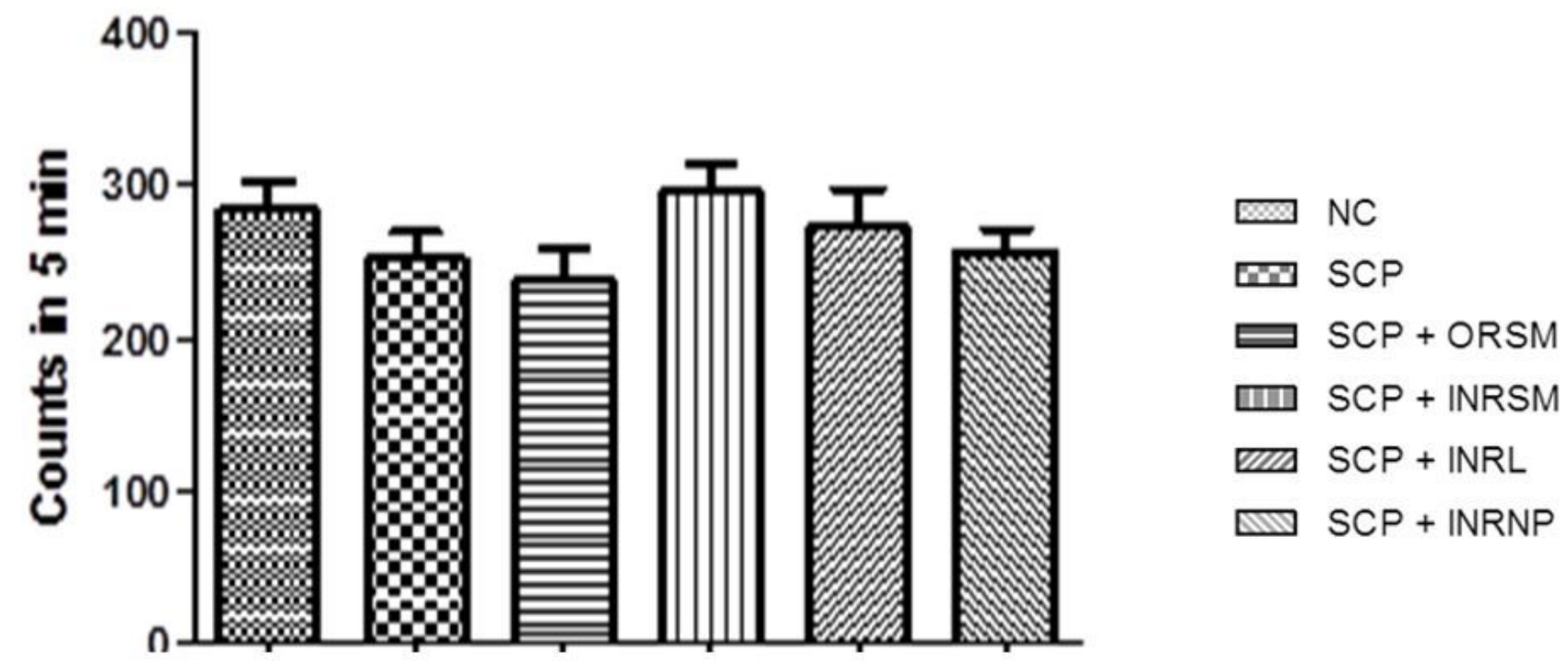

Figure 4

Influence of different formulations of rivastigmine on locomotor activity in scopolamine induced amnesia in rats.

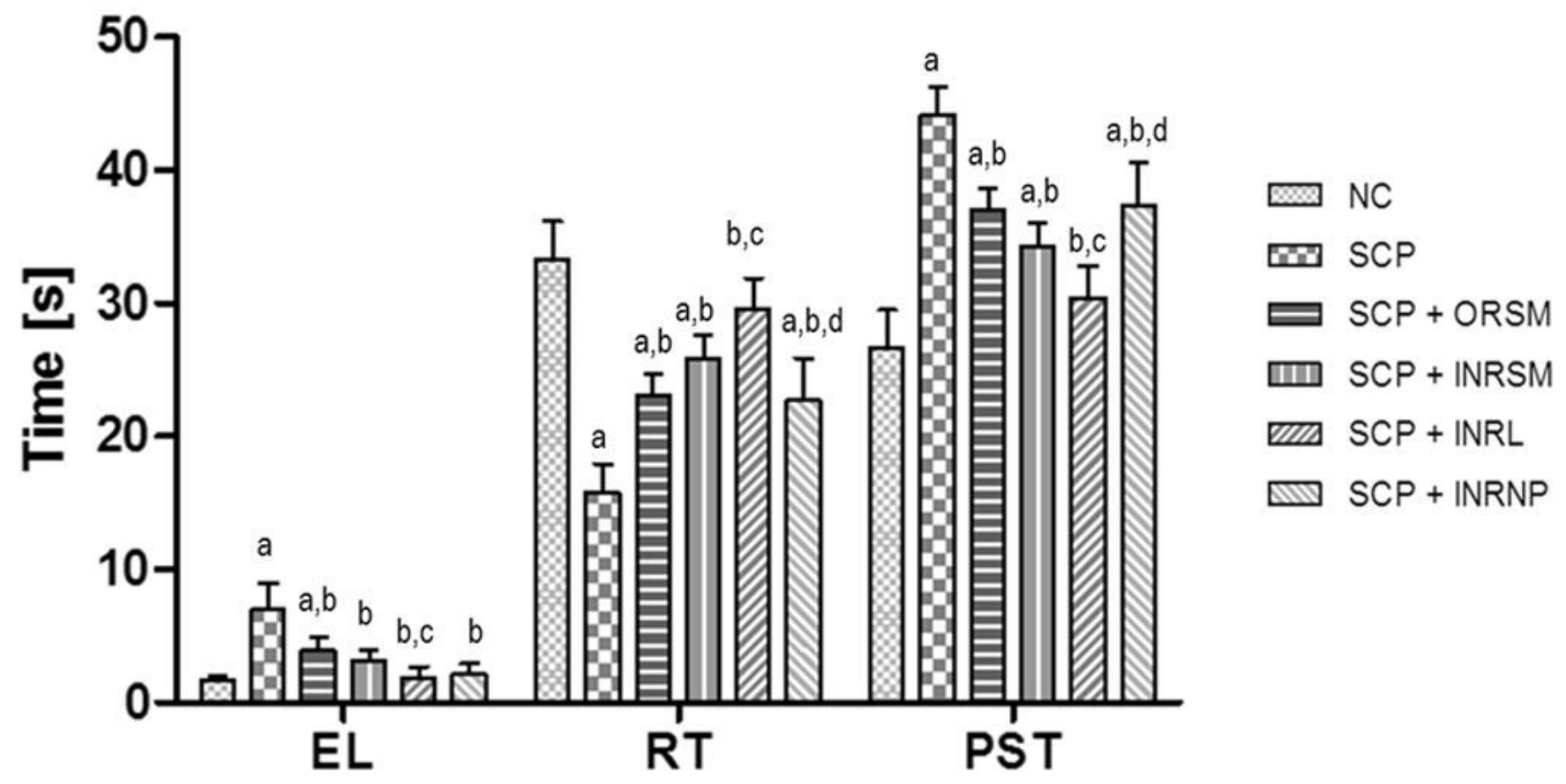

Figure 5 
Influence of different formulations of rivastigmine in scopolamine induced amnesia on MWM task parameters, EL- Escape latency, RT- Residence time and PST- Peripheral swim time). Data represented as mean $\pm S D$, ANOVA followed by post-hoc tests $* a=p<0.05$ vs. NC; ${ }^{*} b=p<0.05$ vs. SCP; ${ }^{*}=p<0.05$ vs. SCP + ORSM; *d $=p<0.05$ vs. SCP + INRL.
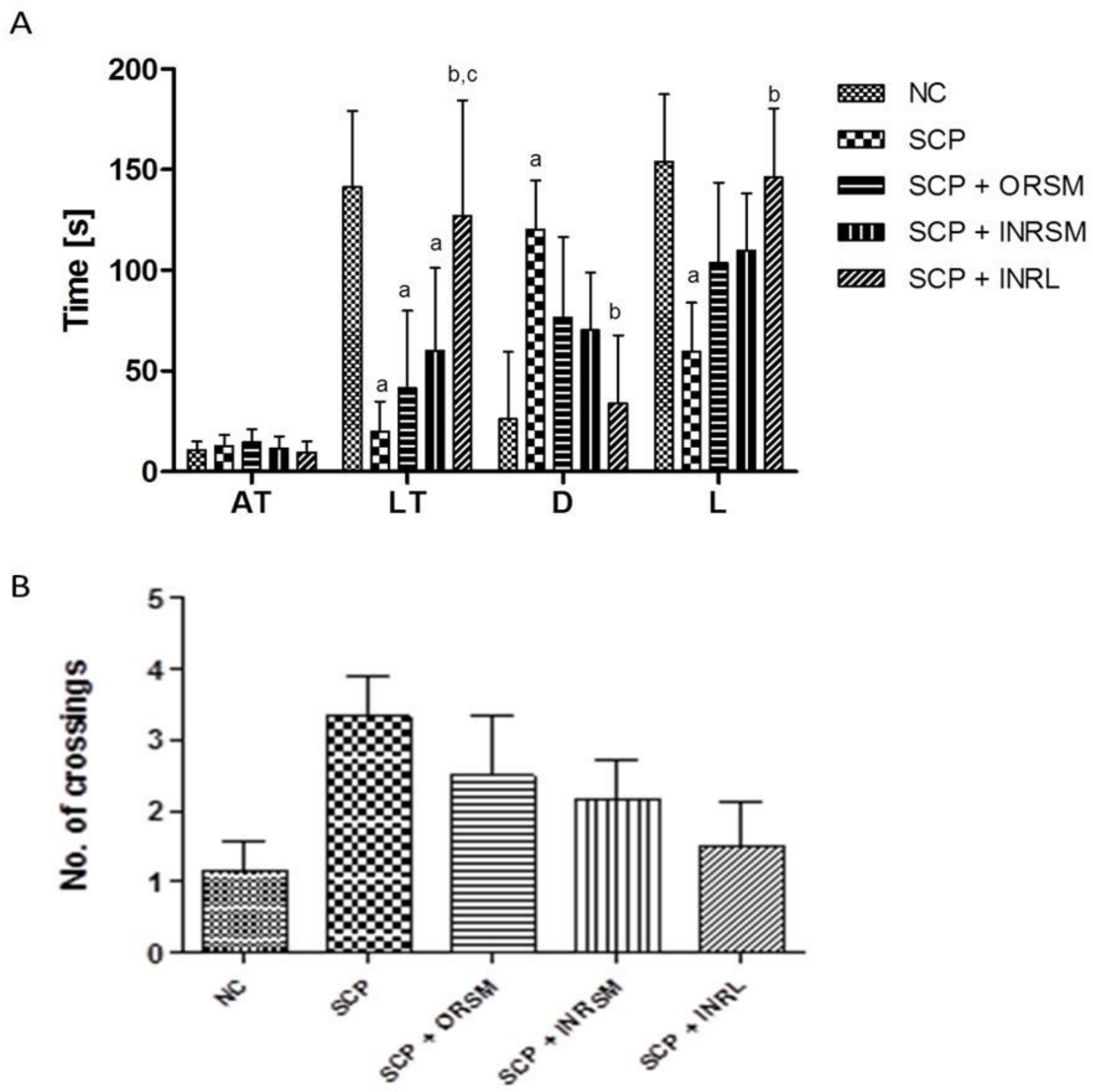

Figure 6

Effect of different rivastigmine formulations administered through different routes of administration in scopolamine induced amnesia on parameters of passive avoidance task. (A) AT (Acquisition time), LT (latency time to enter into darker compartment), D (time spent in the darker compartment), $\mathrm{L}$ (time spent in the brighter compartment) and (B) Number of crossings. Data represented as mean \pm SD, ANOVA 
followed by post-hoc tests ${ }^{*} a=p<0.05$ vs. normal control; ${ }^{*} b=p<0.05$ vs. SCP; ${ }^{*} \mathrm{C}=p<0.05 \mathrm{vs}$. SCP + ORSM.

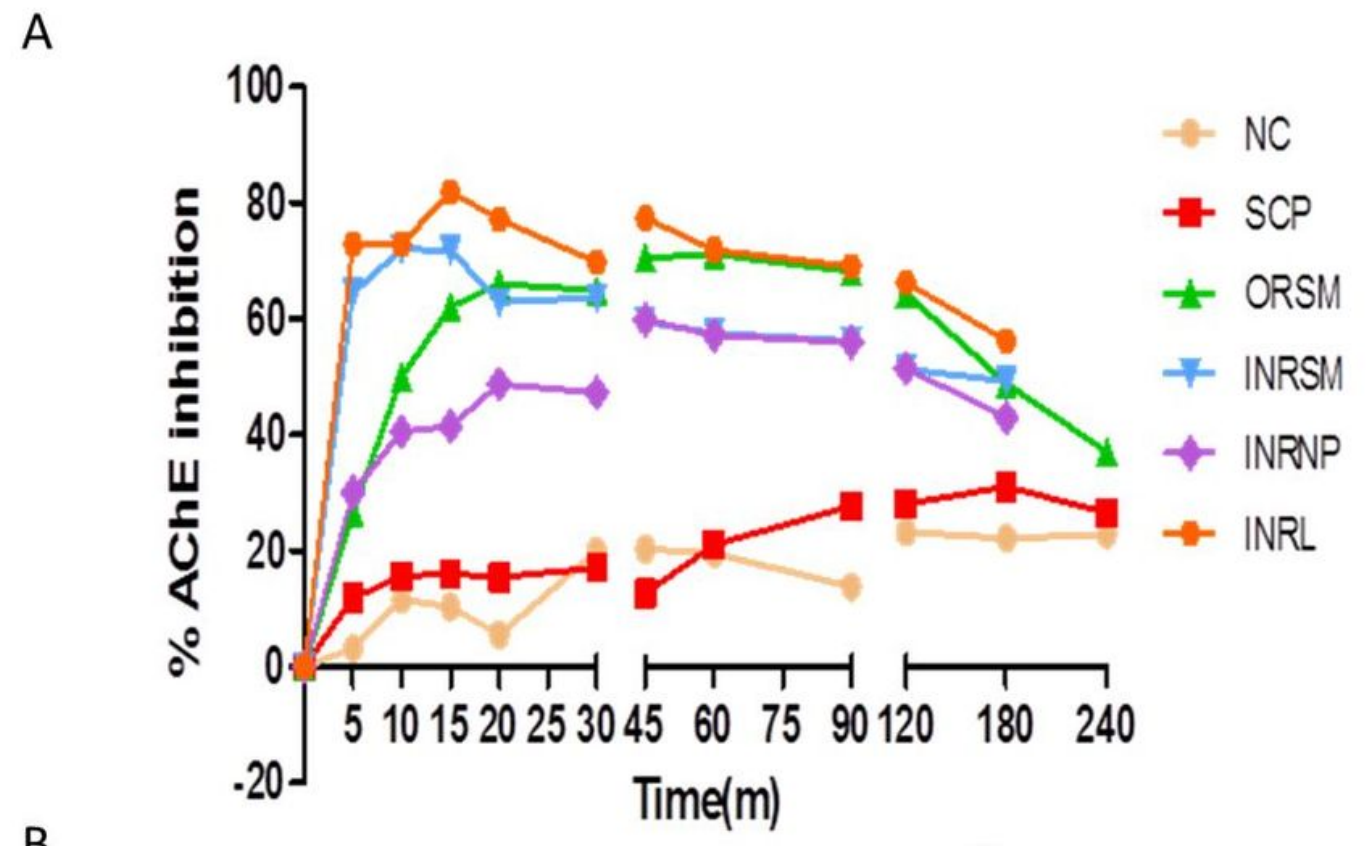

B
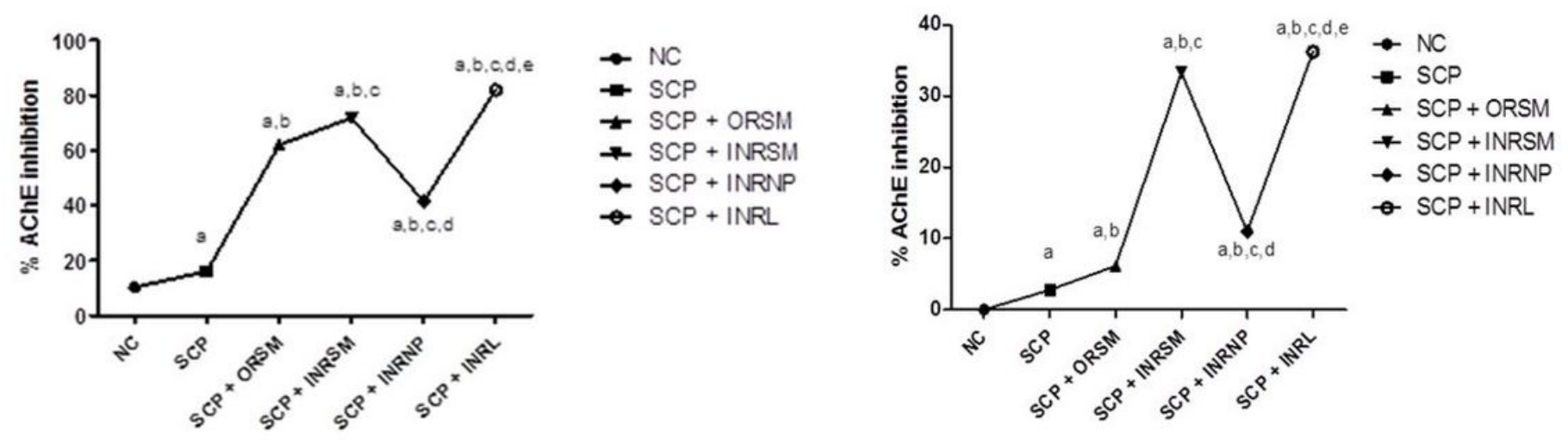

Figure 7

Influence of different rivastigmine formulations on \% AChE inhibition in scopolamine induced amnesic rats. (A) At different time intervals with plasma samples. (B) At $15 \mathrm{~min}$ with plasma samples. (C) At $15 \mathrm{~min}$ with whole brain homogenate. Data represented as mean $\pm S D$, ANOVA followed by post-hoc tests. $* a=p$ $<0.05$ vs. normal control; ${ }^{*} b=p<0.05$ vs. SCP; ${ }^{*} C=p<0.05$ vs. SCP $+O R S M ;{ }^{*} d=p<0.05$ vs. SCP + INRNP; $e=p<0.05$ vs. SCP + INRNP). 


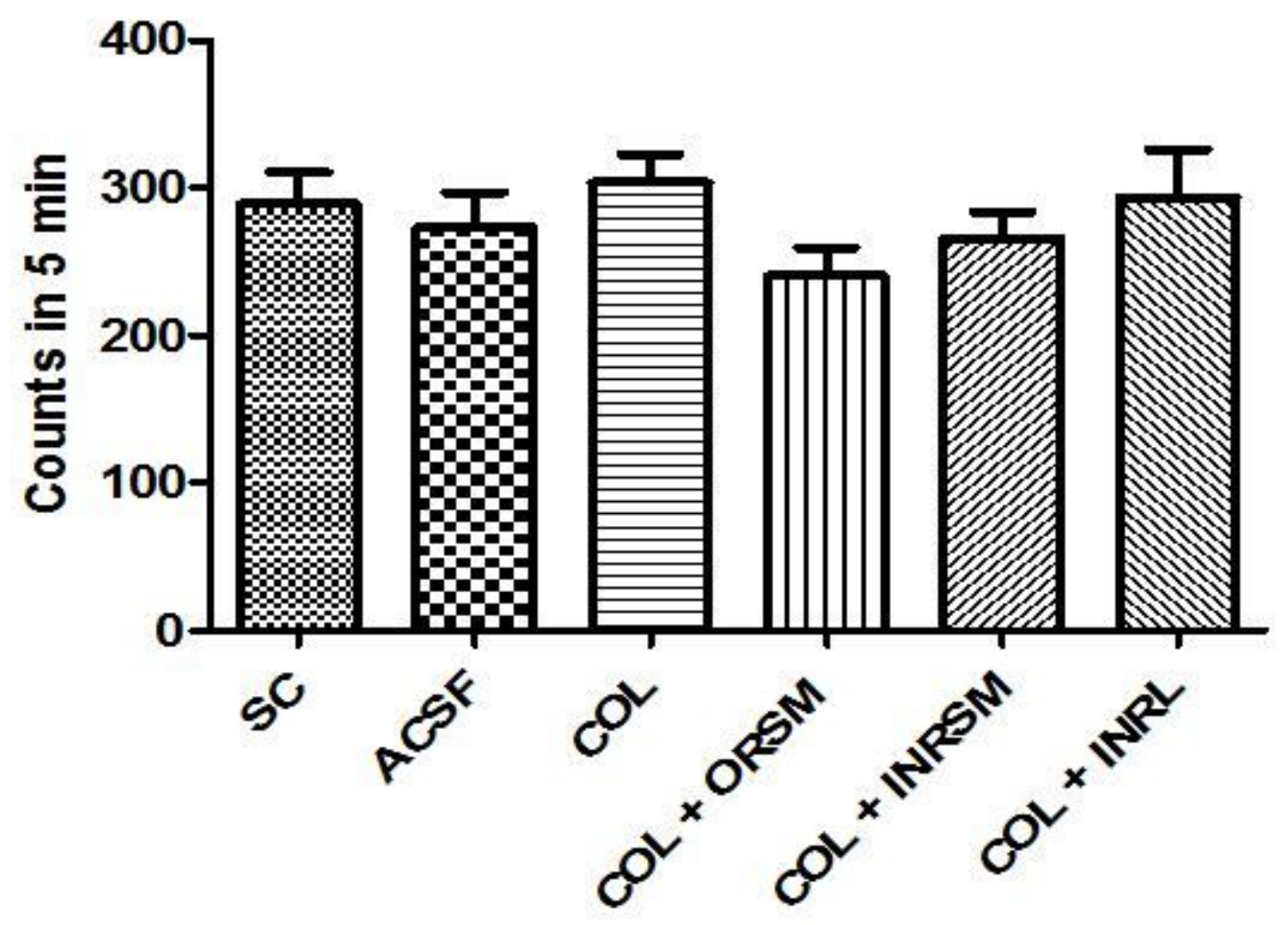

Figure 8

Influence of different rivastigmine formulations on locomotor activity in colchicine induced dementia in rats. 
A
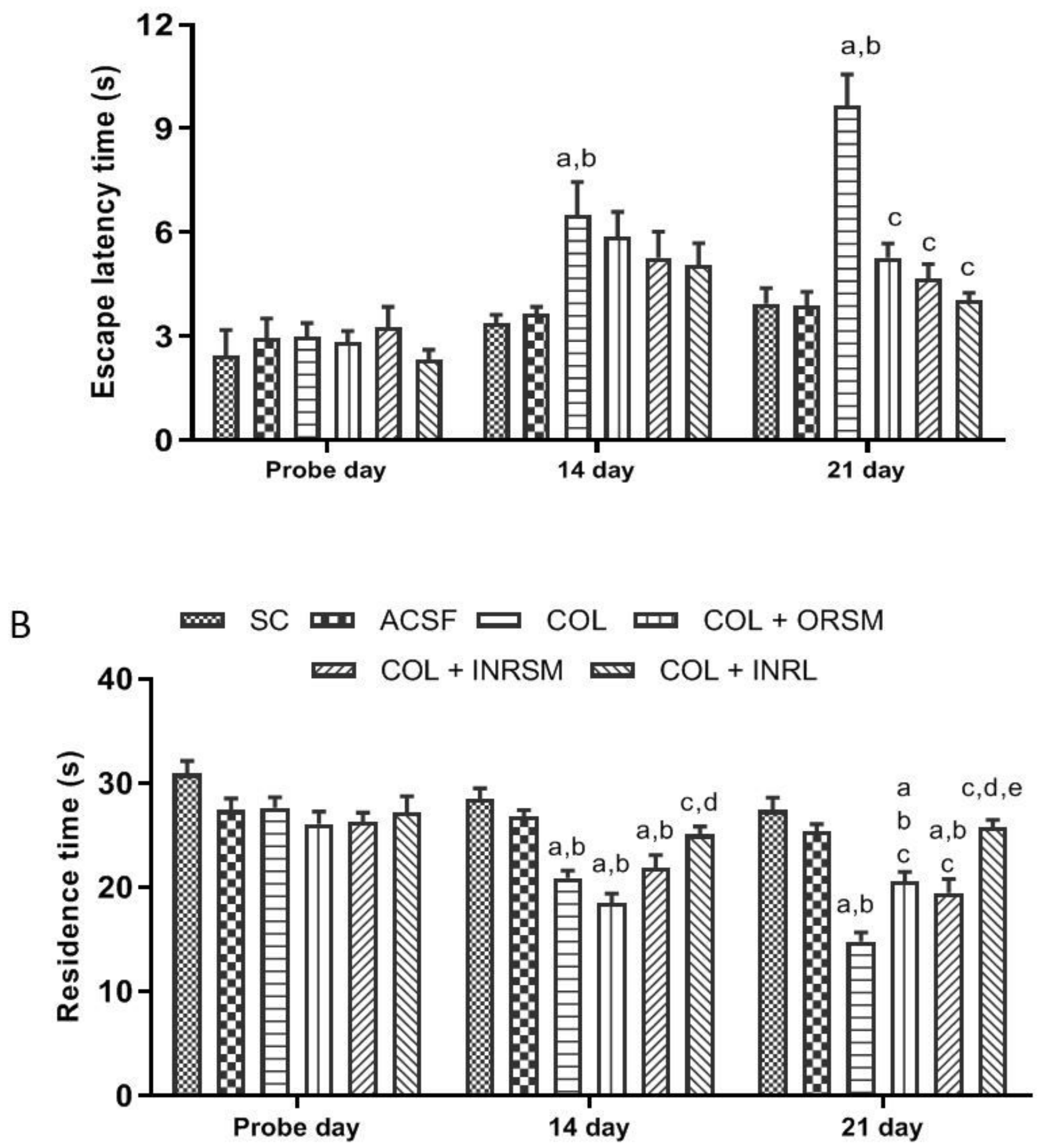

Figure 9

Influence of different rivastigmine formulations in colchicine induced dementia rats assessed by Morris water maze task parameters, A) Escape latency and B) residence time. Data represented as mean $\pm S D$, ANOVA followed by post-hoc tests. ${ }^{*} a=p<0.05$ vs. SC; ${ }^{*} b=p<0.05$ vs. ACSF; ${ }^{*}=p<0.05$ vs. COL; ${ }^{*} d=$ $p<0.05$ vs. COL + ORSM; ${ }^{*}=p<0.05$ vs. COL + INRSM. 

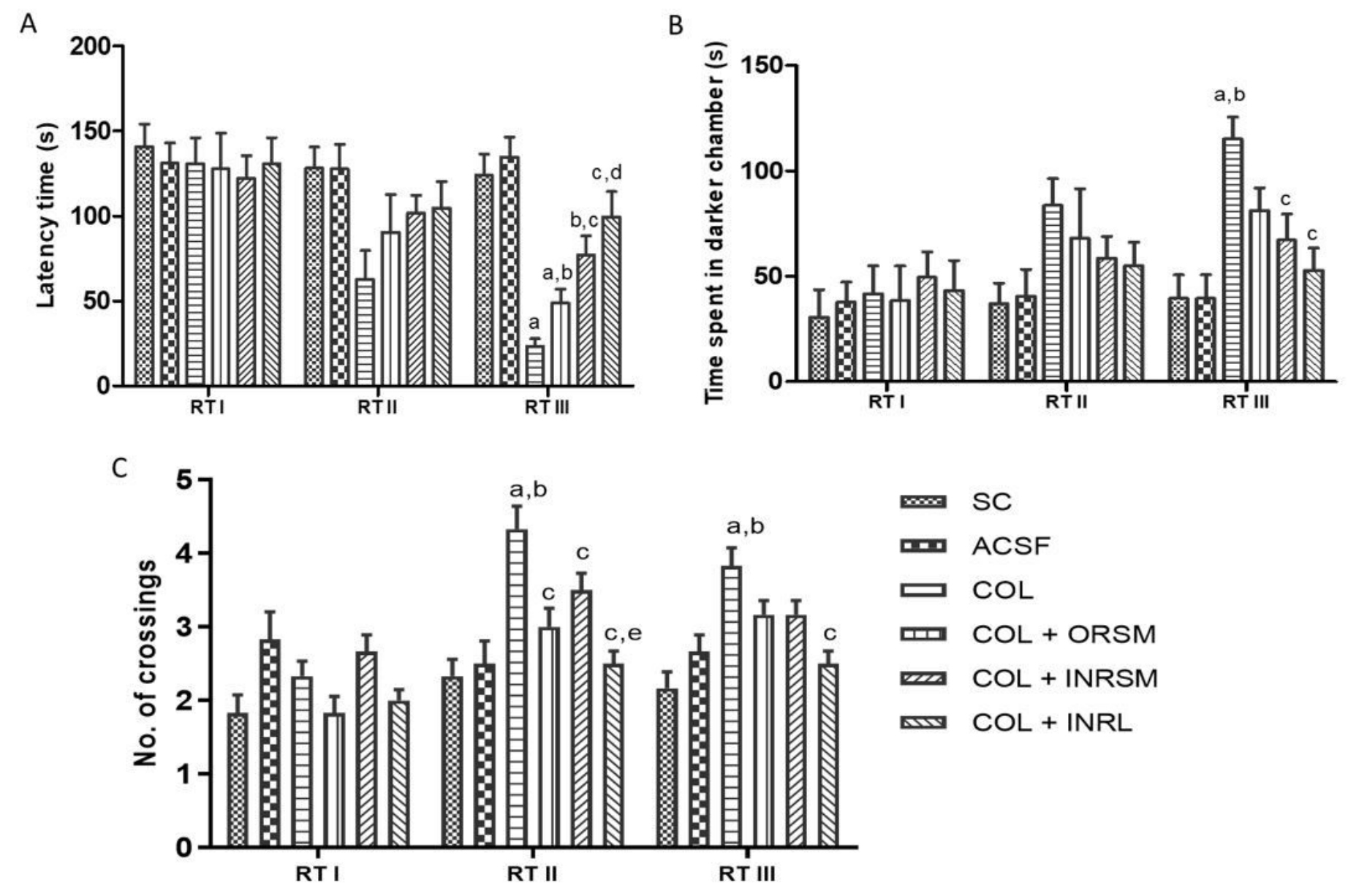

Figure 10

Influence of different rivastigmine formulations on (A) latency time to enter the dark chamber, (B) Time spent in darker chamber, (C) Number of crossings on different retention test days during PAT in colchicine induced dementia rats. Data represented as mean $\pm S D$, ANOVA followed by post-hoc tests. $* a=p<0.05$ vs. SC; ${ }^{* b}=p<0.05$ vs. ACSF; ${ }^{*} \mathrm{C}=p<0.05$ vs. COL; ${ }^{\star} d=p<0.05$ vs. COL $+O R S M ;{ }^{*}=p<0.05$ vs. COL + INRSM. 


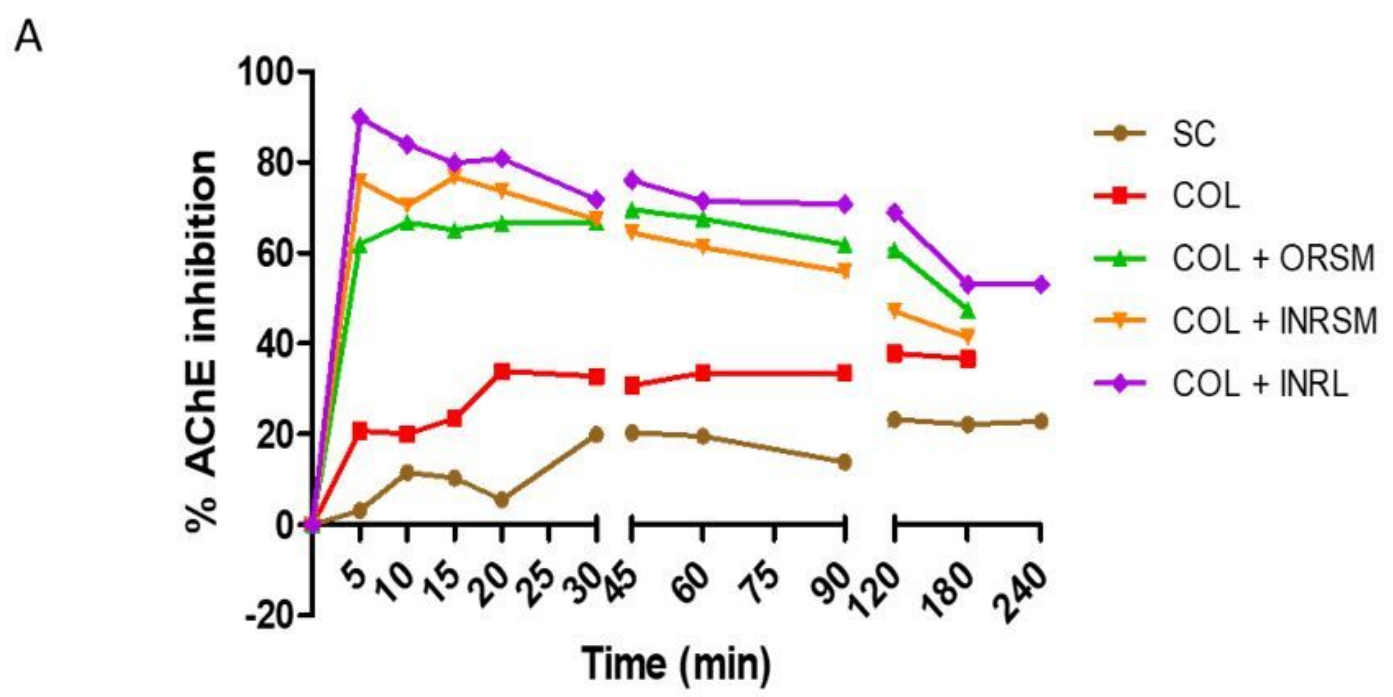

B

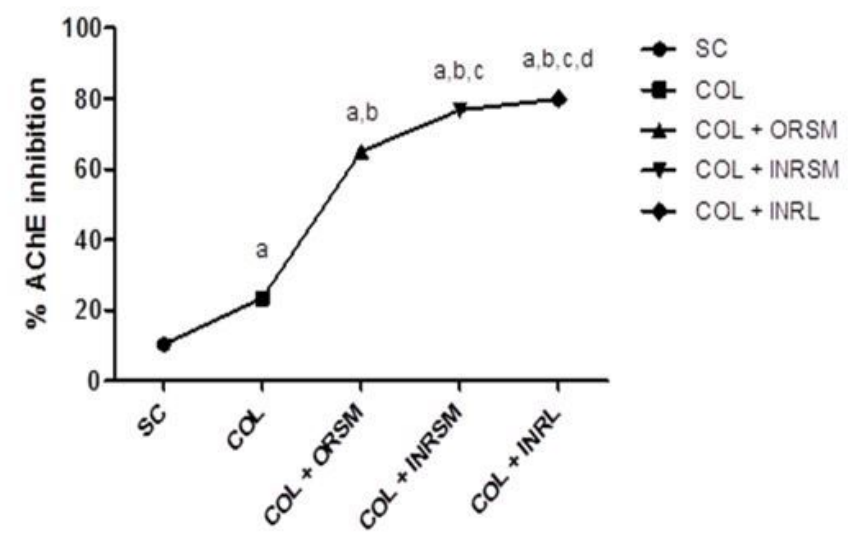

C

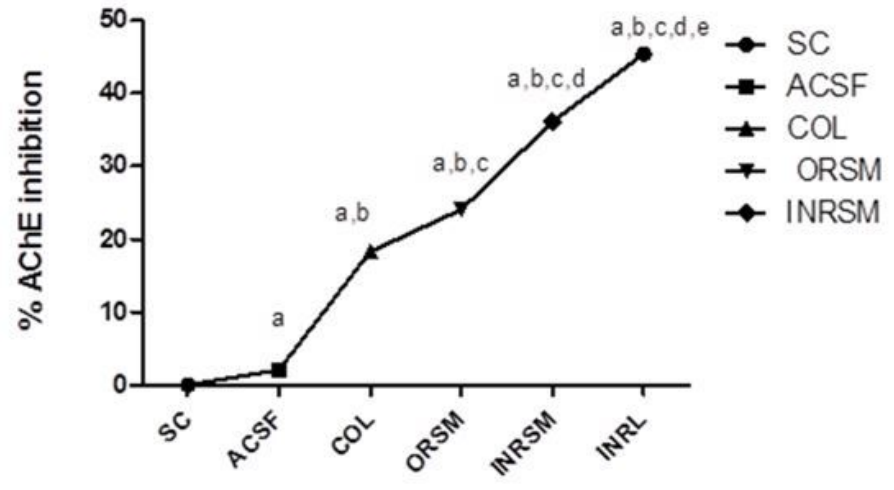

Figure 11

Influence of different rivastigmine formulations on \% AChE inhibition in colchicine induced dementic rats. (A) At different time intervals with plasma samples. (B) At $15 \mathrm{~min}$ with plasma samples. (C) At $15 \mathrm{~min}$ with whole brain homogenate. Data represented as mean $\pm S D$, ANOVA followed by post-hoc tests $\left({ }^{*} a=p<\right.$ 0.05 vs. SC; ${ }^{* b}=p<0.05$ vs. ACSF; ${ }^{*} \mathrm{C}=p<0.05$ vs. COL; ${ }^{*} d=p<0.05$ vs. $C O L+O R S M ;{ }^{*}=p<0.05$ vs. COL+ INRSM). 
A
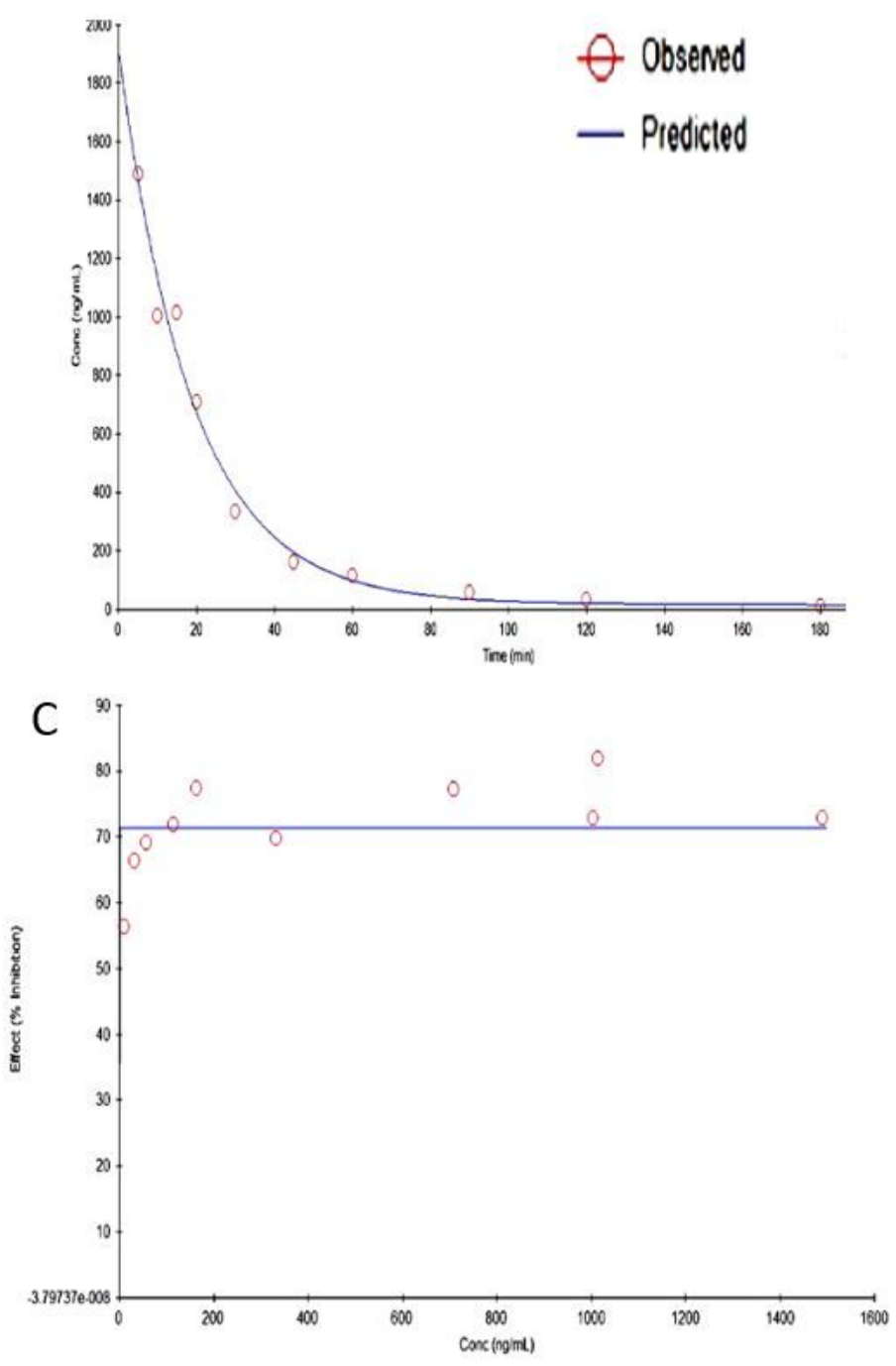

B

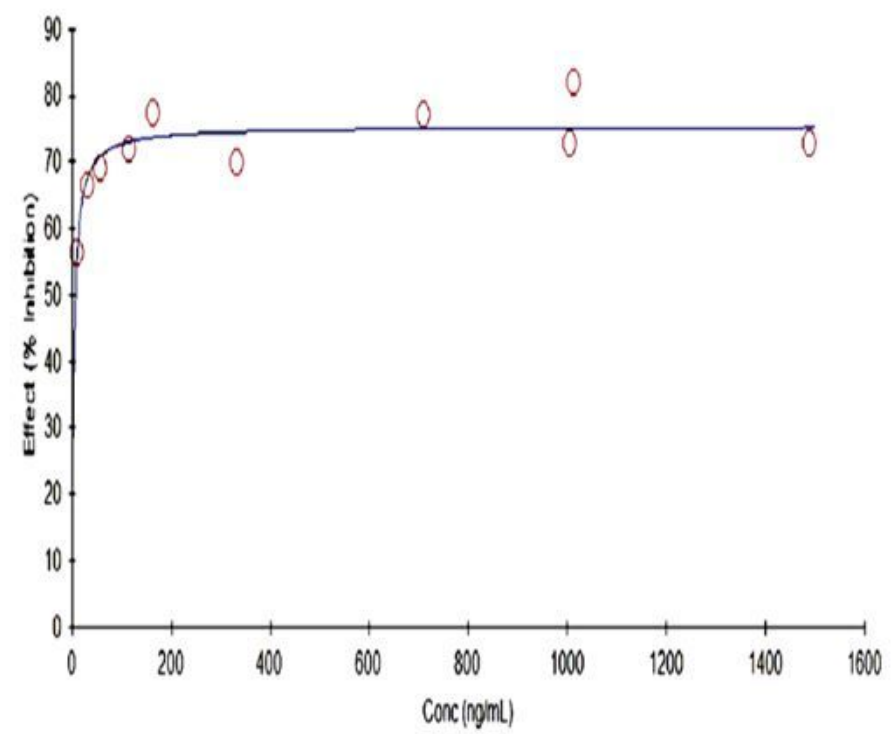

D

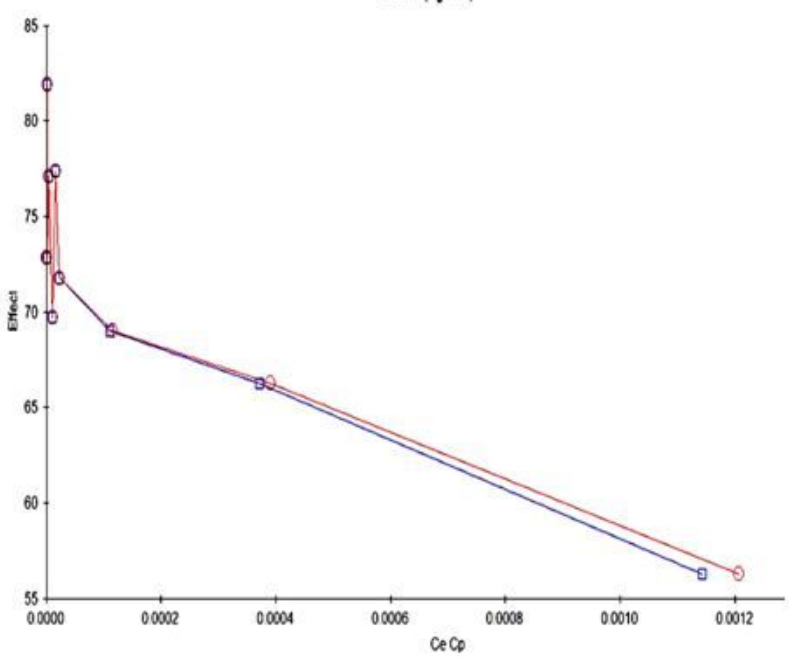

Figure 12

Predicted and observed models of rivastigmine loaded liposomes following intranasal administration in scopolamine induced rats. (A) PK model predicted plasma concentration vs. time relationship (B) PD model predicted plasma concentration vs. AChE inhibition. (C) PK-PD model predicted time vs. plasma AChE inhibition. (D) PK-PD model predicted hysteresis curve.

\section{Supplementary Files}

This is a list of supplementary files associated with this preprint. Click to download.

- DataRSM.xlsx 